\title{
MedienPädagogik
}

Zeitschrift für Theorie und Praxis der Medienbildung

\section{Zur Verankerung von Medienerziehung in den Bildungsplänen für Kindertageseinrichtungen}

Theresa Lienau und Lies van Roessel

\begin{abstract}
Zusammenfassung
Kinder werden heute in eine mediatisierte Welt hineingeboren und ihr Alltag ist von Anfang an durch Medien geprägt. Neben den Eltern kommt auch den frühkindlichen Bildungseinrichtungen die wichtige Aufgabe zu, erste mediale Erfahrungen der Kinder aufzugreifen und sie auf einen souveränen Umgang mit Medien vorzubereiten. In den Bildungs- und Erziehungsplänen, die in den frühen 2000ern deutschlandweit eingeführt wurden, formulieren die Bundesländer Richtlinien für die pädagogische Arbeit in Kindertageseinrichtungen. Ziel der vorliegenden Studie ist es, zu prüfen, ob und wie die aktuellen Bildungspläne das Thema Medien aufgreifen und inhaltlich ausgestalten. Wir zeigen, dass die Bildungspläne hinsichtlich des Umfangs und der Ausgestaltung des Themas Medienerziehung stark variieren: Während manche Pläne es ausführlich und facettenreich behandeln, widmen sich andere dem Thema gar nicht oder nur sehr knapp. In Kontrast zu Friedrichs-Liesenkötter (2019) wird ein methodischer Ansatz vorgeschlagen, der weniger von strukturellen als von inhaltlichen Merkmalen ausgeht: Anhand von Baackes vier Dimensionen der Medienkompetenz (2007) schlüsseln wir zum einen auf, welche Fähigkeiten laut den Plänen in Bezug auf Medien erworben werden sollen. Zum anderen gruppieren wir die Pläne in vier Intensitätsstufen der Verankerung ein und analysieren sie einzeln in Hinblick auf ihre inhaltlichen Schwerpunkte. Die Studie bildet somit eine Grundlage für weitere empirische Forschung nach dem Einfluss der Bildungspläne auf die pädagogische Arbeit in Kindertageseinrichtungen.
\end{abstract}

On the integration of media education in the German early childhood education plans

\begin{abstract}
Children are born into a profoundly mediatised world, in which (digital) media are ubiquitous from the very start. Early childhood education as well as parents have a responsibility to help kids to process first media experiences and prepare them to become confident and informed media users. From the 2000s on, the German federal states have started to formulate their guidelines for pre-school education in education plans (the socalled Bildungs- und Erziehungspläne), as to formalize the German daycare system. The aim of this paper is to analyse whether and how the federal states have integrated media
\end{abstract}


and media education into their current education plans. We will show that the plans vary substantially regarding the scope and form of media education. While some plans address the subject in a detailed and diverse way, others cover the issue only briefly or not at all. In contrast to Friedrichs-Liesenkötter (2019), we suggest a methodological approach that focuses on content rather than form: Using Baacke's four dimensions of media literacy, we have categorized the plans into four levels to illustrate the plans' different depths of the integration of media literacy / media education. The study provides a basis for further empirical research into the influence of the education plans on the daily practices of German daycare institutions.

\section{Einleitung}

Unser Alltag ist zunehmend von Medien geprägt. Nicht nur die Welt der Erwachsenen ist heute tiefgreifend mediatisiert (Hepp 2018), auch die Kleinsten kommen mit diversen Medien - auch digitalen - in Berührung. So umfasst die Mediennutzung von Kleinkindern neben dem noch als Leitmedium geltenden Fernseher mittlerweile auch Smartphones und Tablets. ${ }^{1}$ Studien zum Mediennutzungsverhalten von KitaKindern belegen, dass Medien nicht nur immer häufiger und länger, sondern auch in immer früherem Alter genutzt werden (vgl. Guth 2018; Feierabend et al. 2015, 2017a, 2017b).

Erwachsene - Eltern wie Erzieherinnen und Erzieher - stehen der veränderten Mediennutzung von Kleinkindern oftmals kritisch gegenüber. Teilweise idealisieren sie die eigene Medienkindheit (Fleischer et al. 2018) oder fordern die Kita als medienfreien Schonraum (Eder et al. 2017). Die Medienerfahrungen der Kinder sollten jedoch kein Tabu darstellen, vielmehr ist es eine Aufgabe der Kita als erster Bildungsinstitution, diese in der pädagogischen Arbeit aufzugreifen (e.g. Anfang 2018; Eder et al. 2017; Roboom 2017; Tilemann 2018). Der Ruf nach einer gezielten Medienerziehung bzw. -bildung fusst dabei auf der Erkenntnis, dass Medien als Teil der Lebenswelt der Kinder weitreichenden Einfluss auf deren Entwicklung haben (Eder et al. 2017). Entsprechend wird gefordert, dass Kinder bei ihren frühen Medienerfahrungen pädagogisch begleitet und unterstützt werden, um das entwicklungsfördernde Potenzial von Medien auszuschöpfen und Risiken einzudämmen (e.g. Anfang 2018, Eder et al. 2017, Eggert und Wagner 2016). Aus der UN-Kinderrechtskonvention von 1989, der Sofia-Strategie des Europarats (2016) sowie den darauf aufbauenden «Leitlinien zur Achtung, zum Schutz und zur Erfüllung der Rechte des Kindes im digitalen Umfeld»

1 Laut der jährlich durchgeführten Kinderwelten-Studie liegt der Anteil der 3- bis 5-Jährigen, die täglich Fernseher, Smartphone, Tablet und/ oder Laptop nutzen, im Jahr 2018 bei 74\%. Dabei nimmt die Nutzung mobiler Geräte stetig zu: In 58\% der Haushalte mit Kindern von 3-5 Jahren ist heute ein Tablet vorhanden, welches auch von jedem vierten Kind genutzt wird (Guth 2018). Zum Vergleich: Die Kinderwelten-Studie aus dem Jahr 2015 besagte, dass nur 12\% der 3-5-Jährigen das Tablet nutzen und auch die Verbreitung liegt mit 33\% deutlich niedriger (Guth 2015). 
(2019) lässt sich ableiten, dass Kinder von Geburt an ein Recht auf Medienbildung haben. Diese sollte sich am Entwicklungsstand des einzelnen Kindes orientieren und die Rechte des Kindes auf Teilhabe, Befähigung und Schutz abwägend einbeziehen.

Die Begriffe Medienerziehung und Medienbildung werden teilweise synonym, teilweise jedoch auch abweichend verwendet (vgl. Friedrichs-Liesenkötter 2019). Mit Medienerziehung werden insbesondere bewusste, auf ein medienpädagogisches Ziel ausgerichtete Handlungen gegenüber Kindern beschrieben (vgl. Tulodziecki 1995); sie befasst sich insofern mit der «praktischen pädagogischen Arbeit im Bereich der Medien» (Eder et al. 2013). Die Ausformulierung des angestrebten medienpädagogischen Ziels unterscheidet sich: Die DGfE (Sektion Medienpädagogik, DGfE 2017) beschreibt Medienerziehung als «Anleitung und Unterstützung eines förderlichen, sozial-kommunikativen Medienverhaltens», während Brüggemann et al. (2013) Medienerziehung als "gezielte Förderung eines kompetenten, kritischen, reflektierten, kreativen und verantwortungsvollen Umgangs mit Medien bei den adressierten Kindern» definieren. Tulodziecki (2008) zufolge umfasst der Begriff «alle Aktivitäten und Überlegungen in Erziehung und Bildung [...], die das Ziel haben, ein humanes bzw. verantwortliches Handeln im Zusammenhang mit der Mediennutzung und Mediengestaltung zu entwickeln.». Der Begriff der Medienbildung ist weniger klar definiert, zielt jedoch tendenziell auf ein Verständnis von Bildung als Prozess ab (Jörissen und Marotzki 2009; Spanhel 2010; 2011), in dem die Person «sein ganzes Leben hindurch eine kritische Distanz zu den Medien und ihren Weiterentwicklungen aufbaut und eine Verantwortungshaltung gegenüber den Medien und im Umgang mit ihnen einnimmt» (Spanhel 2002, 6). Jörissen und Marotzki (2009) beschreiben Medienbildung als Bildung in einer Welt, in der Medien omnipräsent sind (einer «mediatisierten» Welt, nach Hepp (2018)), was zu veränderten Selbst- und Weltverhältnissen des Menschen führt.

In den Bildungsplänen werden die Begrifflichkeiten meist nicht klar voneinander getrennt, weshalb in der Analyse generell nach pädagogischen Inhalten in Bezug auf den Bildungsbereich Medien gefragt wird. Medienbildung wird in aktuellen Debatten häufig dem Begriff der Medienkompetenz gegenübergestellt (vgl. Spanhel 2011; Tulodziecki 2010; 2011; Schorb 2009; Aufenanger 1999). Letzterer wird insbesondere dafür kritisiert, dem prozesshaften Medienbildungsbegriff eine erwerbbare Medienkompetenz entgegenzustellen, die eher auf einen Zustand verweist und weniger auf Persönlichkeitsbildungsprozesse als auf inhaltliche Wissensbestände und Fähigkeiten eingeht (vgl. Thomann 2015). Umgekehrt beinhalten Medienkompetenzmodelle wie das von Baacke (2007) auch kritisch-analytische, reflexive oder ethische Elemente (Rosebrock und Zitzelsberger 2002), entsprechen also keinem «funktionaltechnologische[n] Kompetenzverständnis» (Tulodziecki 2011, 22). In der vorliegenden Analyse untersuchen wir die Bildungspläne auch auf die im Zusammenhang mit dem Bildungsbereich Medien zu erwerbenden Kompetenzen; die Vielzahl der 
genannten Fähigkeiten lässt erkennen, dass die Pläne entsprechend kompetenzorientiert ausgerichtet sind.

Dass eine frühe Medienerziehung nicht nur als Aufgabe der Eltern, sondern auch der Kita gesehen wird, ist auch im Zusammenhang mit dem sich wandelnden gesellschaftlichen Bild früher Bildung zu sehen. Die Betreuungsquote von Drei- bis Sechsjährigen in Kindertageseinrichtungen liegt bereits seit 2008 über $90 \%$ und steigt bei den Unter-Dreijährigen sukzessive an (Statistisches Bundesamt 2018). Der Elementarbereich hat längst nicht nur Fürsorge-Pflichten zu erfüllen, sondern wird zunehmend, politisch sowie gesellschaftlich, als erste Bildungsinstitution betrachtet. ${ }^{2}$ So wurden $2004 \mathrm{im}$ neunseitigen «Gemeinsamen[r] Rahmen der Länder für die frühe Bildung in Kindestageseinrichtungen» (JMK und KMK 2004) die Anforderungen der Politik an die frühkindliche Bildung festgeschrieben. Die Debatte wurde angeregt durch das schlechte Abschneiden Deutschlands bei den ersten PISA-, IGLU- und OECD-Studien; auch gab es in vielen anderen OECD-Staaten bereits Pläne für diesen Bildungsbereich (Textor 2019). Die von den Bundesländern schrittweise eingeführten Bildungs- und Erziehungspläne ${ }^{3}$ sollten den Zielen des gemeinsamen Rahmens entsprechen (Betz und Eunicke 2017) und somit einen «Abschied von der Unverbindlichkeit» einläuten (Diskowski 2008, 50). Gemein ist innen das Ziel, Bildungsstandards im frühkindlichen Bereich zu etablieren. Bereits im «Gemeinsamen[r] Rahmen der Länder» umfasst dies auch das Thema Medien:

«Was den Umgang mit Medien angeht, gehört zur Medienkompetenz als dem obersten Ziel von Medienbildung auch die Fähigkeit, Medien zweckbestimmt und kreativ zu nutzen und damit eigene Werke zu erstellen.»

Betz und Eunicke (2017) konstatieren, dass es zu den Bildungsplänen - gemessen an ihrer flächendeckenden Implementierung - bislang nur wenige wissenschaftliche Publikationen gibt (Betz und Eunicke 2017, 5). In Bezug auf Medienerziehung wurden die Pläne jedoch bereits in verschiedenen Publikationen thematisiert (e.g. FriedrichsLiesenkötter 2019; Friedrichs und Meister 2015; Institut für Informationsmanagement Bremen ifib 2015; Kammerl und Thumel 2016; Neuß 2013; Urlen 2018). Hier ist vor allem die Arbeit von Friedrichs-Liesenkötter (2019) hervorzuheben, welche eine systematische Analyse bietet, die auch die aktuellen, überarbeiteten Bildungspläne aller Bundesländer einbezieht (Stand: 01/2019). Die Arbeit entwirft eine dreistufige Kategorisierung der Bildungspläne in Bezug auf die Verankerung von Medienerziehung/

2 Dass die frühkindliche Bildung immer stärker in den Fokus der Politik rückt, zeigt auch das 2019 in Kraft getretene «Gute-Kita-Gesetz».

3 Im Folgenden nennen wir die Bildungs- und Erziehungspläne kurz «Bildungspläne». Eine Sammlung findet sich unter https://www.bildungsserver.de/Bildungsplaene-der-Bundeslaender-fuer-die-fruehe-Bildungin-Kindertageseinrichtungen-2027-de.html. Teilweise werden die Pläne durch Handreichungen und Broschüren ergänzt, welche einzelne Aspekte genauer in den Blick nehmen, etwa die Arbeit mit Kindern von 0 bis 3 Jahren (BE, HE, NI), die Zusammenarbeit mit den Eltern (SH) oder auch einzelne (NI für «Sprachbildung und Sprachförderung») bzw. sämtliche Bildungsbereiche (SH). 
-bildung, die sich jedoch an formalen Kriterien orientiert. Die Kategorien lauten: 1. Verankerung als separater Bildungsbereich bzw. als Bildungsbereich in Koppelung mit anderen Bildungsbereichen («grüne» Kategorie); 2. Medienerziehung/-bildung unter anderen Bildungsbereichen behandelt («gelbe» Kategorie); 3. Medienerziehung/bildung nicht behandelt («rote» Kategorie). Bereits Meister et al. (2012) kamen zu dem Schluss, dass die Einordnung als eigenständiger Bildungsbereich «nicht direkt etwas über die Intensität der Verankerung von Medienerziehung in den Bildungsplänen aus[sagt]». Wir schlagen daher eine Kategorisierung vor, die auf inhaltlichen Kriterien fusst (siehe unten). Mit dieser Vorgehensweise wollen wir den Fokus stärker darauf legen, welches Verständnis von Medienerziehung bzw. -bildung in den einzelnen Bildungspläne vertreten wird und weniger, ob oder wie sie das Thema formal integrieren. Des Weiteren erscheint uns eine Unterscheidung von drei Kategorien als zu grob: Die «grüne» Kategorie bei Friedrichs-Liesenkötter (2019) enthält zehn der 16 Pläne. Daher haben wir uns für ein Vier-Kategorien-System entschieden, welches zwischen einer «fehlenden bis schwachen», «eingeschränkten», «umfassenden» und «sehr umfassenden» Verankerung unterscheidet.

Darüber hinaus soll der Vergleich mit einer früheren Untersuchung der Bildungspläne durch Meister et al. (2012) Aufschlüsse darüber geben, ob von einer Intensivierung der Verankerung des Themas in aktuelleren Plänen gesprochen werden kann. ${ }^{4}$

Ziel der vorliegenden Studie ist es, zu prüfen, ob und wie die aktuellen Bildungspläne das Thema Medien aufgreifen und inhaltlich ausgestalten. Welche Begriffe von Medien, Medienerziehung bzw. -bildung sowie Medienkompetenz verwenden die verschiedenen Bundesländer? Wie ausführlich widmen sie sich dem Thema? Was sind, den Bildungsplänen zufolge, die Ziele der medienerzieherischen Arbeit und welche Schwerpunkte setzen sie dabei? Wir werden zeigen, dass die Bildungspläne hinsichtlich des Umfangs und der Ausgestaltung des Themas Medienerziehung bzw. -bildung stark variieren: Während manche Bundesländer das Thema ausführlich und facettenreich behandeln, widmen sich andere Bildungspläne dem Thema gar nicht oder nur sehr knapp.

Im Folgenden werden wir zunächst die Vorgehensweise erläutern, bevor wir die Ergebnisse der Analyse präsentieren.

4 Bereits Meister et al. (2012) stellten fest, dass die Rolle von Medienerziehung je nach Land und Bildungsplan erheblich variiert. Die Studie bietet einen guten, wenn auch etwas knappen Einblick in die unterschiedlichen Schwerpunkte in Bezug auf Medien und Medienerziehung der Bildungspläne. Der Fokus der Untersuchung lag jedoch auf dem Bildungsplan Nordrhein-Westfalens (NRW) und es wurden nur drei weitere Bildungspläne in Referenz zu NRW analysiert. Zudem wurden seit der Datenerhebung der 2012 veröffentlichten Studie zehn Bildungspläne in veränderter Neuauflage veröffentlicht. Dies betrifft die Bildungspläne von Bayern (2016), Berlin (2014), Hamburg (2012), Hessen (2014), NRW (2016), Sachsen (2011), Saarland (2018), Sachsen-Anhalt (2013) Schleswig-Holstein (2012), Thüringen (2015). 


\section{Vorgehensweise}

Gegenstand der Analyse waren die aktuellen Bildungspläne (Stand: 10/2019) aller 16 Bundesländer. Um die Textstellen, die sich mit dem Thema Medien beschäftigen, zu identifizieren, wurden alle Pläne systematisch durchsucht und die entsprechenden Textstellen dokumentiert. ${ }^{5}$ Einige Pläne setzen nicht nur für den Elementarbereich Richtlinien fest. So beziehen sich Hessen, Mecklenburg-Vorpommern und NRW auf die Arbeit mit Kindern von 0-10 Jahren und Thüringen auf die Altersspanne von 0-18 Jahren. Aufgrund dieser Divergenzen und um eine bessere Vergleichbarkeit zu gewährleisten, wurden die Aussagen, die eindeutig auf die Arbeit mit älteren Kindern und Jugendlichen eingehen, in der Analyse nicht berücksichtigt. Des Weiteren bezieht sich die Analyse ausschliesslich auf Medienerziehung bzw. -bildung, d.h. nicht auf den Einsatz von Medien in der Kita für andere Aufgaben, etwa für Öffentlichkeitsoder Portfolioarbeit. Auch haben wir Textstellen ausgeschlossen, die explizit nur auf den Umgang mit Büchern und anderen analogen Medien eingehen.

Zunächst haben wir geprüft, wie sich die Bildungspläne hinsichtlich der Verankerung des Medienthemas im Inhaltsverzeichnis, der Umfänglichkeit der entsprechenden Textstellen sowie den darin genannten Begrifflichkeiten und Zielsetzungen unterscheiden. Anschliessend haben wir die Kompetenzen, die laut den Bildungsplänen im Kontext mit Medien in der Kita vermittelt werden sollen, anhand der vier Dimensionen des Medienkompetenzmodells nach Baacke kategorisiert. Dafür wurden alle genannten Kompetenzen identifiziert und den entsprechenden Dimensionen (Medienkritik, -kunde, -nutzung und -gestaltung) zugeordnet. Wir haben aufgeschlüsselt, welche Kompetenzen in den Plänen besonders häufig auftauchen und somit aus Sicht der Bildungspläne zentral für die frühkindliche Bildung sind.

Darüber hinaus diente uns diese Einordnung nach Baackes Modell dazu, auszuwerten, wie intensiv das Thema Medien in den Plänen verankert ist. Die Pläne wurden sowohl einzeln hinsichtlich ihrer inhaltlichen Schwerpunkte analysiert als auch miteinander in Bezug gesetzt, um übergreifende Tendenzen sichtbar zu machen.

\section{Medien in den Bildungsplänen: Eine erste Einordnung}

\section{Allgemeines: Umfang, Aktualität und Verbindlichkeit}

Die Bildungspläne der Bundesländer weisen starke Unterschiede hinsichtlich Seitenzahl, Erscheinungsdatum und auch ihrer Verbindlichkeit für die Praxis auf. So variiert die Länge der Bildungspläne zwischen 33 (BB) und 505 (BY) Seiten. Damit ist der Bayerische Plan der mit Abstand umfangreichste, gefolgt von Thüringen (376 S.) und Mecklenburg-Vorpommern (296 S.).

5 Die hierfür verwendeten Suchbegriffe waren «Medien $»$, , «medial ${ }^{\star} »$, «digital ${ }^{\star} »$ und «elektronisch"». 
Auch die Erscheinungsdaten der Pläne sind sehr unterschiedlich. Während die neuesten Bildungspläne aus den Jahren 2018 (SL), 2016 (BY, HE und NW) bzw. 2015 (TH) stammen, gibt es noch immer vier Bundesländer, in denen die Pläne seit den Nuller-Jahren nicht verändert wurden (HB und RP: 2004, NI: 2005 und BB: 2006). Die restlichen Pläne (BE, BW, HH, MV, SH, SN, ST) wurde im Zeitraum von 2010-2014 veröffentlicht. ${ }^{6}$

Auch bezeichnen sich die Bildungspläne in unterschiedlichem Mass als verbindlich. Die meisten Pläne sind eher als «Orientierungsrahmen», «Leitfaden», «Empfehlungen» oder «Anregungen» angelegt, es gibt jedoch auch einige, in denen ihr verbindlicher Charakter betont wird. Im Vorwort zum Bremer Plan heisst es beispielsweise, dass «Bremen zu den ersten Bundesländern [gehörte], das verbindliche Grundsätze der frühkindlichen Bildungs- und Erziehungsarbeit beschrieben hat.»In Mecklenburg-Vorpommern wird der Plan - etwas widersprüchlich - als «verbindliche Orientierung zur Gestaltung der Bildungsprozesse» beschrieben. Auch der schleswigholsteinische Plan sagt: «Die weiterentwickelten Bildungsleitlinien [...] formulieren die Ziele, denen sich Kindertageseinrichtungen inzwischen verpflichtet sehen [...].» In Bayern ist zwar der Bildungsplan selbst als Orientierungsrahmen gedacht, die darin formulierten Bildungs- und Erziehungsziele sind jedoch auch gesetzlich in der Ausführungsverordnung zum BayKiBiG festgehalten worden und als solche verbindlich. Auf die Verbindlichkeit der Bildungspläne und deren Konsequenzen für die Praxis werden wir in der Diskussion (siehe unten) eingehen.

\section{Verankerung des Themas Medien in den Inhaltsverzeichnissen}

Wie bereits von Friedrichs-Liesenkötter (2019) dargelegt, zeigt sich bereits in den Inhaltsverzeichnissen der Pläne, dass das Thema Medien bzw. Medienbildung/ -erziehung nicht in allen Plänen die gleiche Aufmerksamkeit erfährt: Während es in einigen Plänen einen eigenständigen Bildungsbereich ausmacht, taucht es andernorts gar nicht auf. Insgesamt führen zehn Pläne das Thema im Inhaltsverzeichnis auf, davon drei als eigenständigen Bildungsbereich (NW, RP, TH). In den sieben weiteren Plänen wird Medienbildung bzw. -erziehung als Teilbereich aufgeführt, meist in Verbindung mit dem übergeordneten Bildungsbereich Sprache/ Kommunikation/ Sprechen bzw. Schrift (BY, BE, HH, HE, SN, SL). In Schleswig-Holstein wird das Thema hingegen mit musisch-ästhetischer Bildung in Zusammenhang gebracht. In den übrigen Plänen taucht das Thema im Inhaltsverzeichnis nicht auf (BB, BW, HB, MV, NI, ST).

Im Vergleich mit den Ergebnissen von Meister et al. (2012), die ebenfalls die Inhaltsverzeichnisse der Pläne hinsichtlich des Medienthemas angeschaut haben,

6 Die Pläne von Bremen, Niedersachsen und Rheinland-Pfalz wurden in unveränderter Fassung (HB, RP) bzw. als Gesamtausgabe mit den ergänzenden Handlungsempfehlungen, jedoch in unveränderter Form (NI) zu einem späteren Zeitpunkt neu herausgegeben (HB: 2012, NI: 2018, RP: 2016). 
lassen sich nur vereinzelt Veränderungen beobachten. So besagt die Studie, dass im damaligen thüringischen Plan Medienbildung nicht aufgeführt wurde; der aktuelle Plan beinhaltet es hingegen als eigenständigen Bildungsbereich. Im hessischen Plan wurden Medien damals als eigener Bereich behandelt, nun wird es unter das Thema «Kommunikationsfreudige und medienkompetente Kinder» gefasst. Im Falle des schleswig-holsteinischen Plans wurde Medienbildung zum Stand der 2012er-Studie in Verknüpfung zu Kommunikation, Schrift und Sprache gesehen, heute hingegen mit «Musisch-ästhetische Bildung und Medien - oder: sich und die Welt mit allen Sinnen wahrnehmen» betitelt.

In drei Plänen (die seit der Datenerhebung durch Meister et al. (2012) nicht aktualisiert wurden) taucht das Thema nicht im Inhaltsverzeichnis auf, wird jedoch schwerpunkthaft unter einem anderen Bildungsbereich behandelt (BW, MV, NI). Medienbildung wird hier unter die Bildungsbereiche «Sinne» (BW), «Kommunikation, Sprechen und Sprache(n)» (MV) bzw. «Ästhetische Bildung» (NI) gefasst. Die Verbindungen zu anderen Bildungsbereichen sind ein Indiz dafür, welche Medienkompetenzen KitaKinder den Plänen zufolge erwerben sollten. Die häufige Nähe zum kommunikativsprachlichen Bereich ist im Kontext damit zu sehen, dass der Medienbegriff in den meisten Plänen auch klassische Druckmedien umfasst und diese teilweise auch besonders hervorgehoben werden. Darüber hinaus wird der Einsatz von technischen Geräten wie etwa Computern oder Tablets in der Kita häufig kritisch gesehen (e.g. Eder 2018; Institut für Demoskopie Allensbach 2014), weshalb eine stärkere Verknüpfung zu technischen Themen möglicherweise zu Ablehnung führen würde. Der Bezug zu sinnlichen oder ästhetischen Erfahrungen zeugt insofern von einem weniger auf Technik fokussierten Verständnis von Medienpädagogik und den darüber zu erwerbenden Kompetenzen.

Die in den Plänen gesetzten Schwerpunkte (Sprache, Sinne, Ästhetik) sind vor dem Hintergrund der pädagogischen Arbeit mit Kindern im Kita-Alter zu verstehen, während bei älteren Kindern, Jugendlichen und auch Erwachsenen in der Regel andere Fokusse gesetzt werden.

\section{Umfang des Bereichs Medien}

Wie viel Raum dem Thema Medien in den Plänen zugemessen wird, ist ebenfalls sehr unterschiedlich. Die längsten und ausführlichsten Kapitel zum Thema Medien finden sich im bayerischen und thüringischen Bildungsplan, die sich auf je etwas über 20 Seiten mit dem Thema auseinandersetzen. Dabei ist jedoch zu beachten, dass der thüringische Plan für Kinder und Jugendliche von 0 bis 18 Jahre ausgelegt ist, während sich der bayerische nur auf die Zeit vor dem Schuleintritt bezieht. Andere Pläne widmen sich dem Thema Medien (fast) gar nicht (insbesondere BB, HB, ST). 
Schaut man sich den proportionalen Anteil des Kapitels zur Medienbildung im Vergleich zum Gesamtumfang des jeweiligen Plans an, zeigt sich folgendes Bild: Hamburg widmet dem Thema einem Anteil von etwas über 6\% der Gesamtseitenzahl, gefolgt von Schleswig-Holstein, Thüringen, Bayern und NRW, die es auf 4-6\% ihres Gesamtumfangs behandeln. Der Rest der Pläne beschäftigt sich jedoch auf unter 3\% ihrer Gesamtseitenzahl mit Medien, Medienbildung und/oder Medienkompetenz. Auffällig ist, dass die fünf Pläne, die dem Thema prozentual gesehen am meisten Raum beimessen, neueren Datums und in den Jahren zwischen 2012 und 2016 erschienen sind.

\section{Verwendung des Medienbegriffs}

Es zeigt sich, dass die Pläne mit sehr unterschiedlichen Medienbegriffen arbeiten und diese häufig undifferenziert verwenden. So zeugt eine Aufzählung wie «unterschiedliche[n] Medien (Papier, verschiedene Schreibgeräte, Schreibmaschine/ Computer)» (BB) nicht von einem differenzierten Medienverständnis. Im saarländischen Plan werden Medien als «Mittler aller Art» beschrieben: «Bücher, Erzählungen, Bilder in Büchern, auf Werbeplakaten, Filme, Spielzeug ...» Nur in wenigen Plänen werden verschiedene Medien voneinander unterschieden und digitale Medien auch explizit als solche benannt (BY, NW, TH und im Ansatz BE). Im bayerischen und thüringischen Plan wird auch auf Medienkonvergenz eingegangen. In einigen Plänen überwiegt hingegen ein eher klassisches, nicht auf digitale Medien ausgerichtetes Medienverständnis (BW, BB, HB, SL, MV, ST) und insbesondere im mecklenburg-vorpommerschen Plan wird die Bedeutung von (Vor-)Lesemedien betont.

Wenn sich die Bildungspläne auf digitale Medien beziehen, ist damit meist der Computer / PC gemeint. Mobile Medien wie Tablets oder Smartphones werden lediglich in dem 2018 erschienenen saarländischen Plan erwähnt, in den anderen Plänen hingegen (wenn überhaupt) nur, um die Alltagsrelevanz dieser Geräte zu betonen. Es wird jedoch in keinem Plan näher erläutert, wie z.B. Tablets für die pädagogische Arbeit mit Kita-Kindern genutzt werden können. Dies ist insofern verwunderlich, als das Schlagwort «Medienpädagogik in der Kita» im medialen und gesellschaftlichen Diskurs häufig mit dem Einsatz von Tablets in Verbindung gebracht wird. Teilweise sind die Pläne jedoch auch so alt, dass die Medienlandschaft zum Erscheinungsdatum noch eine andere und mit anderen Herausforderungen verknüpfte war.

Einige Pläne versuchen, die negativen Haltungen gegenüber digitalen Medien zu adressieren und zu entkräften, indem sie digitale mit analogen oder elektronischen (nicht-digitalen) Medien vergleichen. So adressiert der rheinland-pfälzische Plan, der für sein Erscheinungsdatum im Jahr 2004 (er ist damit der älteste noch aktuelle Plan) vergleichsweise progressiv mit dem Thema Medien umgeht, die oftmals skeptischen Haltungen: «Reiht man sie [die Medien der modernen Technik] in die grosse Vielfalt der in Kindertagesstätten unhinterfragt akzeptierten Medien ein, ist es möglich, 
damit verbunden Fragen offen zu begegnen und für die Praxis in den Einrichtungen fruchtbar zu machen.» Auch der (deutlich aktuellere, 2016 erschienene) bayerische Plan betont: «Alle Medien beinhalten Chancen und Risiken. Es gibt nicht die «guten» und die «schlechten» Medien.» Des Weiteren wird ausgeführt: «Die gedruckte Information ist nicht per se seriöser als die in Bild und Ton präsentierte, der Umgang mit Computer und Internet ist nicht per se bildender als der mit dem Fernsehen.» Im saarländischen Bildungsplan findet sich die konfrontative Formulierung: «Eine medienfreie Kita schützt vielleicht die pädagogische Fachkraft vor unbequemen Auseinandersetzungen. Die Kinder schützt es nicht.».

\section{Definitionen von Medienkompetenz und Ziele medienpädagogischer Arbeit}

In den Bildungsplänen der Länder wird häufig nicht definiert, was unter Medienkompetenz zu verstehen ist. Eine Ausnahme sind der bayerische und der hessische Plan (beide von Prof. Dr. mult. Fthenakis verfasst), die Medienkompetenz wortgleich als «bewussten, kritisch-reflexiven, sachgerechten, selbstbestimmten und verantwortlichen Umgang mit Medien» beschreiben. Auch die Ziele medienerzieherischer Arbeit in der Kita werden häufig nicht klar festgelegt. Es zeigen sich hier jedoch unterschiedliche Tendenzen. Erneut formulieren der bayerische und der hessische Plan übereinstimmend: «Medienbildung und -erziehung zielt darauf ab, Risiken entgegenzuwirken, die Orientierungskompetenz zu stärken, die positiven Potentiale nutzbar zu machen sowie der Ungleichverteilung von medienbezogenen Chancen und Risiken entgegenzuwirken.» Im NRW-Plan hingegen steht die Interaktion zwischen Mensch und Medien im Vordergrund. Das Ziel früher Medienbildung sei es, Medien «verantwortungsvoll einzuschätzen und entwicklungsfördernd einzusetzen». Der rheinland-pfälzische Plan stellt dem selbstbestimmten Umgang einem unkritischen Konsum entgegen. Medienerziehung erfolge mit dem Ziel, "Kinder auf einen souveränen Umgang mit Medien sowie einer kritischen [sic!] Haltung hinsichtlich des Medienkonsums vorzubereiten.» Der saarländische sowie der schleswig-holsteinische Plan hingegen heben die Bedeutung realer, sinnlicher Erfahrungen hervor. So heisst es in ersterem, dass Kinder durch medienpädagogische Arbeit lernen können, «Medienerlebnisse mit ihren realen Erfahrungen zu vergleichen und Traum, Fiktion und Lebenswirklichkeit zu unterscheiden.» Und Schleswig-Holstein setzt als Ziel, «eine aktive, viel-sinnige Auseinandersetzung mit Medien [zu] unterstützen». Den in den Plänen genannten Zielen von Medienerziehung liegen also sehr unterschiedliche Wertvorstellungen zugrunde.

Nachdem diese erste Einordnung gezeigt hat, dass die Bildungspläne sowohl hinsichtlich allgemeiner Parameter als auch in Hinblick auf Kategorisierung, Begrifflichkeiten und Zielsetzungen des Medienthemas grosse Unterschiede aufweisen, werden wir im Folgenden die Pläne tiefer gehend anhand von Baackes Medienkompetenzmodell analysieren. 


\section{Inhaltliche Ausgestaltung von Medienkompetenz}

Um die Verankerung des Medienthemas in den einzelnen Plänen zu bewerten, haben wir mit dem Kompetenzmodell nach Baacke (2007) gearbeitet, welches vier Dimensionen von Medienkompetenz beschreibt: Medienkritik, Medienkunde, Mediennutzung und Mediengestaltung. In einigen Bildungsplänen wird Medienkompetenz sehr nah an dem Modell beschrieben, weshalb der Rückgriff auf dieses für die Analyse von Medienkompetenz in dem gegebenen Kontext sinnvoll erscheint. ${ }^{7}$

Wir werden im Folgenden darauf eingehen, mit welchen Kompetenzen die jeweiligen Dimensionen in den Plänen ausformuliert werden (dabei ist anzumerken, dass die Dimensionen nicht immer trennscharf voneinander zu unterscheiden sind) und somit zeigen, welche Fähigkeiten im Umgang mit Medien den Plänen zufolge bereits in der Kita erworben werden können und welche davon besonders häufig genannt werden. Entsprechend betrachten wir hier alle Bildungspläne als Gesamtkorpus, während wir weiter unten auf die einzelnen Pläne eingehen und zeigen, wo Medienkompetenz nicht oder nur schwach, eingeschränkt, umfassend bzw. sehr umfassend behandelt wird.

Im Zusammenhang mit der Dimension Medienkritik ${ }^{8}$ ist in den Bildungsplänen häufig von einem kritisch-reflexiven Umgang mit Medien und Medieninhalten die Rede. So heisst es in den Plänen, Medienbeiträge sollen kritisch hinterfragt werden, Medienbotschaften und -tätigkeiten kritisch reflektiert werden oder ein kritisches Bewusstsein soll gegenüber Medien und Medienprodukten erlangt werden. Diese Fähigkeit wird oft in Zusammenhang mit der etwas konkreter formulierten Kompetenz gestellt, dass Kinder lernen, Werbung und ihre Absichten zu erkennen und zu reflektieren. Ebenfalls häufig genannt werden die Fähigkeiten, Medien, ihr Wesen und Medienbotschaften zu durchschauen sowie Medien nach persönlichen Massstäben zu bewerten bzw. sich eine Meinung zu Medien bilden. ${ }^{9}$ Es werden also eher analytische und reflexive Kompetenzen genannt, während ethische Aspekte unerwähnt bleiben. Eine Erklärung dafür wäre, dass letztere nicht dem Entwicklungsstand von Kita-Kindern entsprächen. Dass das Erkennen von Werbung besonders häufig genannt wird, weist darauf hin, dass die Bildungspläne Medienbildung in der Kita oft in Verbindung mit einem konsumkritischen Ansatz sehen.

7 Auch Meister et al. haben die Verankerung von Medienerziehung in den Bildungsplänen anhand von Baackes Dimensionen bewertet, wenngleich in knapper Form und ohne nähere methodische Erläuterung.

8 Baacke formuliert in Hinblick auf die Dimension «Medienkritik» drei Unterdimensionen. Erstens nennt er die analytische, welche besagt, dass Hintergrundwissen zur Medienlandschaft notwendig ist, um problematische Medienentwicklungen kritisch und differenziert zu betrachten. An zweiter Stelle führt er die reflexive Unterdimension auf, nach der eigenes (Medien-)Handeln kritisch reflektiert werden soll. Die dritte ist die ethische Unterdimension, welche sowohl auf die analytische und die reflexive Unterdimension bezogen ist und sie mit sozialer Verantwortung verknüpft.

9 Weniger häufig genannte Punkte lassen sich der Tabelle 1 entnehmen. 
Unter der Dimension Medienkunde ${ }^{10}$ - dem Wissen über heutige Medien und Mediensysteme - taucht in den Bildungsplänen besonders häufig auf, dass die Kinder Fähigkeiten zur Nutzung bzw. Bedienung von Medien ${ }^{11}$ erwerben sollten. Dies ist die insgesamt - also unter allen vier Dimensionen - am häufigsten erwähnte Kompetenz; hier liegt also ein besonderer Schwerpunkt. Beispiele, die in diesem Zusammenhang genannt werden, sind etwa: Sender auswählen/ wechseln, Software starten, Grundkenntnisse im Umgang mit Schreibgeräten/ Computern/ Tablets/ Smartphones erwerben, einen Kassettenrecorder einschalten, Erfahrungen mit Foto- und Videokamera machen, Informationen im Internet suchen oder Bilder am Computer malen. Ferner wird in vielen Plänen angeregt, dass Kinder lernen sollten, Medien als Mittel zur Informationsbeschaffung zu verstehen oder zu nutzen. Darüber hinaus wird ein Umgang mit Medien gefordert, der als selbstbestimmt, souverän, selbstständig, eigenverantwortlich, konstruktiv, kompetent oder sachgerecht beschrieben wird. Die Pläne betonen also, dass sich Kinder in der aktiven Nutzung von Medien als eigenständig und kompetent erfahren. Als weiteren Punkt fordern einige Pläne, dass Kinder auch Wissen über Medientechnik oder die Funktionsweise von Medien ${ }^{12}$ erwerben sollten. Es wird also sowohl die Vermittlung klassischer Wissensbestände, als auch - besonders häufig - Bedienungskompetenzen gefordert. Die Wissensaneignungsfunktion von Medien wird besonders in den Vordergrund gestellt; andere Funktionen (etwa die Nutzung von Medien zur Unterhaltung) tauchen weniger auf.

Unter der Dimension Mediennutzung ${ }^{13}$ haben wir hauptsächlich Kompetenzen erfasst, die mit der Verarbeitung der Medienerfahrungen zu tun haben. Von einer solcher Verarbeitung von Medienerfahrungen und -erlebnissen sowie den damit verbundenen Emotionen ist in den Plänen sehr häufig die Rede. Es heisst zum Beispiel, Medienerlebnisse sollten emotional und verbal verarbeitet werden, man sollte sich mit den Medienerfahrungen der Kinder als Teil ihrer Lebenswirklichkeit auseinandersetzen und die Fachkräfte sollten die Kinder dabei unterstützen, die mit Medien verbundenen Gefühle, Erlebnisse und Fantasien zu verarbeiten. Im Zusammenhang damit wird häufig eine (inter)aktive Auseinandersetzung mit Medien(inhalten)

10 Baacke unterscheidet hier zwei Punkte: Zum einen nennt er die informative Unterdimension, welche klassische Wissensbestände umfasst. Die zweite Unterdimension ist die instrumentell-qualifikatorische, welche die Fähigkeit beschreibt, die entsprechenden Geräte bedienen zu können.

11 Nutzungs- und Bedienungsfähigkeiten sind insbesondere bei digitalen (mobilen) Medien kaum voneinander zu trennen, daher sind die hier genannten Fähigkeiten an der Schnittstelle zwischen den Dimensionen Medienkunde und -nutzung einzuordnen. Insbesondere bei digitalen Medien ist aufgrund der Interaktivität kaum zwischen der reinen Bedienung von Medien, welche unter Medienkunde beschrieben wurde, und deren Nutzung im Sinne einer rezeptiv-wahrnehmenden Erfahrung zu unterscheiden.

12 Weniger häufig genannte Punkte lassen sich der Tabelle 1 entnehmen.

13 Auch die Dimension Mediennutzung unterteilt Baacke in zwei Punkte. Zum einen umfasst dies die rezeptiv-anwendende Unterdimension, die davon ausgeht, dass auch als passiv geltende Tätigkeiten wie etwa Fernsehen aktiv sind und die Verarbeitung solcher Medienerfahrungen erlernt werden muss; es bedarf also entsprechender Nutzungs- bzw. Rezeptionskompetenzen. Zweitens umfasst die Dimension Mediennutzung interaktives Handeln; diese Tätigkeit geht über den ersten Unterpunkt hinaus. 
gefordert, um Medieninhalte und -erfahrungen zu verstehen, zu diskutieren oder über andere Wege (Gesang, Musik, Kunst, Rollenspiel) auszudrücken. Auch sprechen viele Pläne an, dass das bewusste Wahrnehmen von Medien und genaues Hinsehen und Hinhören mit den Kindern geübt werden sollte. ${ }^{14}$

Die in den Bildungsplänen angesprochenen mediengestalterischen ${ }^{15}$ Kompetenzen umfassen hauptsächlich eine aktive, produktive und kreative Mediennutzung. Darunter fällt, dass Kinder erfahren, dass man mit Medien auch selbst produktiv sein kann, etwa indem sie Medien selbst herstellen oder anders mit Medien kreativ tätig werden. Als Beispiele werden Bilder- und Fotogeschichten, Hörspiele, oder einfache Videofilme genannt. Ausserdem sollen Kinder lernen, Medien für eigene Anliegen zu nutzen: sowohl als Ausdrucks- und Gestaltungsmittel, als auch als Kommunikations- und Interaktionsmittel. Die von Baacke auch unter Mediengestaltung gefasste gesellschaftliche Dimension spielt in der frühkindlichen Bildung insgesamt eine geringe Rolle. Vielmehr liegt der Fokus darauf, den Kindern zu vermitteln, dass sie Medien für ihre eigenen Zwecke nutzen können und damit selbst aktiv und kreativ werden können. ${ }^{16}$

Übergreifend haben wir festgestellt, dass zehn Bildungspläne auf alle vier Dimensionen eingehen; dies zeigt, dass Baackes Modell zentral für die Ausformulierung von Medienkompetenz sind. Dabei ist Medienkunde die am häufigsten genannte Dimension (in 13 Plänen), gefolgt von Mediennutzung und -gestaltung (jeweils 12), während elf Pläne Medienkritik als Teil von Medienkompetenz betrachten.

In Bezug auf die einzelnen Fähigkeiten wurde die Nutzungs- und Bedienungskompetenz von Medien (als Komponente der Dimension Medienkunde) am häufigsten genannt. Ebenfalls in zehn oder mehr Plänen wurde ein kritischer bzw. reflexiver Umgang (Medienkritik), sowie die Verarbeitung von Medienerfahrungen und -erlebnissen (Mediennutzung) gefordert. Mit Blick auf die Tabelle 1 ist auffällig, dass die Fähigkeiten, die besonders häufig (in zehn oder mehr Plänen) genannt werden, eher allgemein und übergreifend sind (und sich eng an Baackes Dimensionen orientieren), während sich insbesondere in der Gruppe «in 1-3 Plänen genannt» das Spektrum in diverse Einzelfähigkeiten ausdifferenziert. Hier werden etwa Kompetenzen wie «Mögliche Handlungsalternativen beim Medienumgang mitbedenken», «Ein Verständnis von Fernsehgewalt entwickeln» oder «Kinder dokumentieren ihren Alltag als Speicher biografischer Erfahrungen» aufgeführt.

14 Weniger häufig genannte Punkte lassen sich der Tabelle 1 entnehmen.

15 Die Dimension Mediengestaltung bezieht sich auf die ständige Veränderung von Medien, sowohl technisch als auch inhaltlich. Die erste Unterdimension umfasst Veränderungen bzw. Weiterentwicklung des Mediensystems innerhalb der angelegten Logik und erfordert damit «innovative» Fähigkeiten, während sich die zweite, «kreative» Unterdimension auf gestalterisch-ästhetische Aspekte und die Partizipationskompetenz bezieht. Gestaltung wird demnach nicht nur auf subjektiv-individualistischer Ebene verstanden, sondern auch auf gesellschaftlicher Ebene: als Diskurs der Informationsgesellschaft.

16 Weniger häufig genannte Punkte lassen sich der Tabelle 1 entnehmen. 


\begin{tabular}{|c|c|c|c|c|}
\hline & Medienkritik & Medienkunde & Mediennutzung & Mediengestaltung \\
\hline $\begin{array}{l}\text { in } 10 \text { oder mehr } \\
\text { Bildungsplänen } \\
\text { genannt }\end{array}$ & $\begin{array}{l}\text { Kritischer / reflexiver Um- } \\
\text { gang mit Medien(inhalten) }\end{array}$ & $\begin{array}{l}\text { Fähigkeiten zur Nutzung } \\
\text { / Bedienung von Medien } \\
\text { erwerben }\end{array}$ & $\begin{array}{l}\text { Verarbeitung von Medie- } \\
\text { nerfahrungen und - erleb- } \\
\text { nissen sowie der damit } \\
\text { verbundenen Emotionen }\end{array}$ & \\
\hline $\begin{array}{l}\text { in } 7 \text { - } 9 \text { Bil- } \\
\text { dungsplänen } \\
\text { genannt }\end{array}$ & $\begin{array}{l}\text { Werbung und ihre Absich- } \\
\text { ten erkennen }\end{array}$ & $\begin{array}{l}\text { Medien als Mittel zur In- } \\
\text { formationsbeschaffung } \\
\text { verstehen / nutzen } \\
\text { Selbstbestimmter / sou- } \\
\text { veräner / selbstständiger } \\
\text { / eigenverantwortlicher } \\
\text { / konstruktiver / kompe- } \\
\text { tenter / sachgerechter } \\
\text { Umgang mit Medien } \\
\end{array}$ & $\begin{array}{l}\text { (Inter)aktive Auseinan- } \\
\text { dersetzung mit Medien im } \\
\text { Gespräch, Rollenspiel, der } \\
\text { Kunst etc. } \\
\text { Medien bewusst wahrneh- } \\
\text { men / genaues Hinsehen } \\
\text { und Hinhören }\end{array}$ & $\begin{array}{l}\text { Aktive / produktive Medi- } \\
\text { ennutzung, Medien selbst } \\
\text { herstellen } \\
\text { Kreative Mediennutzung }\end{array}$ \\
\hline $\begin{array}{l}\text { in } 4 \text { - } 6 \text { Bil- } \\
\text { dungsplänen } \\
\text { genannt }\end{array}$ & $\begin{array}{l}\text { Medien, ihr Wesen und } \\
\text { Medienbotschaften durch- } \\
\text { schauen } \\
\text { Bewertung von Medien } \\
\text { nach persönlichen Maß- } \\
\text { stäben / sich eine Meinung } \\
\text { über Medien bilden }\end{array}$ & $\begin{array}{l}\text { Medientechnik / Funk- } \\
\text { tionsweise von Medien } \\
\text { verstehen }\end{array}$ & & $\begin{array}{l}\text { Medien als Ausdrucks- und } \\
\text { Gestaltungsmittel nutzen } \\
\text { Medien als Kommunika- } \\
\text { tions- / Interaktionsmittel } \\
\text { nutzen }\end{array}$ \\
\hline $\begin{array}{l}\text { in } 1 \text { - } 3 \\
\text { Bildungsplänen } \\
\text { genannt }\end{array}$ & $\begin{array}{l}\text { Trennen von Realität, } \\
\text { Fiktion und Virtualität / } \\
\text { von eigenem Erleben und } \\
\text { Medienproduktionen } \\
\text { Reflexion von klischee- } \\
\text { haften Darstellungen in } \\
\text { Medien (etwa in Bezug auf } \\
\text { Geschlecht) } \\
\text { Erkennen, dass Medien } \\
\text { Bedürfnisse beeinflussen / } \\
\text { manipulieren } \\
\text { Medien(inhalte) als vom } \\
\text { Menschen gemacht er- } \\
\text { kennen } \\
\text { Gefahren des Missbrauchs } \\
\text { kennen } \\
\text { Analysieren, wo Medien } \\
\text { zum Einsatz kommen } \\
\text { Auf kritische Haltung } \\
\text { hinsichtlich des Medien- } \\
\text { konsums vorbereiten } \\
\text { Verantwortlicher Umgang } \\
\text { mit Medien } \\
\text { Mögliche Handlungsalter- } \\
\text { nativen beim Medienum- } \\
\text { gang mitbedenken }\end{array}$ & $\begin{array}{l}\text { Medien als Kommunikati- } \\
\text { onsmittel verstehen und } \\
\text { nutzen } \\
\text { Mediensysteme kennen } \\
\text { Erweitertes Verständnis } \\
\text { von Medien / Medienviel- } \\
\text { falt kennenlernen } \\
\text { Medienbetriebe kennen- } \\
\text { lernen } \\
\text { Medien für andere Anlie- } \\
\text { gen nutzen (zur Unterhal- } \\
\text { tung / Entspannung / zum } \\
\text { ästhetischen Erleben) }\end{array}$ & $\begin{array}{l}\text { Sich in der Vielfalt der } \\
\text { Medienwelten orientieren } \\
\text { können / Orientierungs- } \\
\text { kompetenz } \\
\text { Welterfahrung medial } \\
\text { ergänzen, neue Räume } \\
\text { erschließen } \\
\text { Insbesondere Kinder mit } \\
\text { schweren motorischen } \\
\text { Störungen oder Lähmun- } \\
\text { gen erleben über tastenbe- } \\
\text { dienbare Spiel- und Lern- } \\
\text { geräte, dass sie Einfluss } \\
\text { auf ihre Umwelt nehmen } \\
\text { können } \\
\text { Medienerlebnisse ge- } \\
\text { nießen } \\
\text { Ein Bewusstsein für Me- } \\
\text { dienrituale entwickeln } \\
\text { Ein Verständnis von Fern- } \\
\text { sehgewalt entwickeln } \\
\text { Kinder zum rezeptiven } \\
\text { Umgang mit Medien be- } \\
\text { fähigen }\end{array}$ & $\begin{array}{l}\text { Medienprojekte durch- } \\
\text { führen } \\
\text { Medien für eigene Anliegen } \\
\text { / Themen nutzen } \\
\text { Medieninhalte verändern } \\
\text { «Produktcharakter» von } \\
\text { Medien kennenlernen } \\
\text { Kinder dokumentieren } \\
\text { ihren Alltag als Speicher } \\
\text { biografischer Erfahrungen } \\
\text { Explorativer und experi- } \\
\text { menteller Umgang mit } \\
\text { Medien } \\
\text { Den Prozess der «Aneig- } \\
\text { nung von Welt» unter } \\
\text { Einbeziehung von Medien } \\
\text { aktiv gestalten }\end{array}$ \\
\hline
\end{tabular}

Tab. 1.: Häufigkeit der Nennung einzelner Medienkompetenzen in den Bildungsplänen, kategorisiert nach Baacke (2007). 


\section{Intensität der Verankerung von Medienerziehung in den einzelnen Bildungsplänen}

Wie intensiv sich die einzelnen Bildungspläne mit dem Thema Medien auseinandersetzen, variiert vom Auslassen des Themas bis hin zu einer sehr umfassenden Auseinandersetzung. Die oben genannte Studie von Meister et al. (2012) unterscheidet lediglich zwischen einer umfassenden Verankerung sowie einer eingeschränkten bzw. "eher schwachen ${ }^{17}$ Verankerung; Friedrichs-Liesenkötter (2019) arbeitet mit einer Einordnung in drei Kategorien nach der Logik einer Ampel (grün/gelb / rot), die uns jedoch nicht nuanciert genug scheint (in die grüne Kategorie fallen 10 der 16 Pläne). Darüber hinaus wurden die Pläne anhand des formalen Kriteriums der Einordnung des Bereichs Medien im Inhaltsverzeichnis kategorisiert. Die vorliegende Analyse folgt hingegen einer differenzierteren Einordnung, die sich zudem an inhaltlichen Kriterien orientiert und umfangreich erläutert und kommentiert wird.

Wir haben die Intensität der Verankerung zunächst daran festgemacht, ob und inwiefern sich die Bildungspläne mit den Dimensionen nach Baacke beschäftigen. Eine fehlende bis schwache Verankerung weisen die Bildungspläne auf, die keine oder nur eine der Dimensionen von Baacke behandeln. Werden zwei oder drei der Dimensionen behandelt, wird die Intensität der Verankerung als eingeschränkt beschrieben. Wenn alle vier Dimensionen angesprochen werden, gehen wir von einer umfassenden Verankerung aus. Wir sprechen von einer sehr umfassenden Verankerung, wenn nicht nur alle vier Dimensionen angesprochen, sondern auch ausführlich behandelt werden und ein breites und gut fundiertes Verständnis von Medienkompetenz bzw. Medienerziehung erkennbar ist.

Fehlende bis schwache Verankerung

Die Pläne der Bundesländer Brandenburg, Sachsen-Anhalt und Bremen weisen eine fehlende bis schwache Auseinandersetzung mit dem Thema Medien auf.

Brandenburg und Sachsen-Anhalt sind die einzigen Bundesländer, die keine Kompetenz nennen, die einer der vier Dimensionen zugeordnet werden kann. Im Brandenburger Plan werden in Bezug auf Medien lediglich «Papier, verschiedene Schreibgeräte [und] Schreibmaschine/Computer» genannt, um eine Schreibecke auszustatten; an anderer Stelle ist von dem Einsatz eines Tonbands und Kassettenrekorders die Rede.

Auch im Plan von Sachsen-Anhalt spielen Medien praktisch keine Rolle, bis auf den Satz: «Schrift wird [von Kindern] über Medien wie Fernsehen und Zeitung wahrgenommen, an Lebensmitteln und Fahrzeugen gesehen und als Leuchtreklame in Geschäften oder in Büchern mit spannenden Geschichten entdeckt.»

17 Da bei Meister et al. (2012) nicht erläutert wird, inwiefern die «eingeschränkte» bzw. «eher schwache» Verankerung zu unterscheiden sind, werden diese hier zusammengefasst. Die eindeutigen Zuordnungen finden sich in Tabelle 2. 
Der Bremer Plan geht ebenfalls nur sehr vereinzelt auf Medien ein. Er problematisiert den «übermäßigen Mediengebrauch», der aus der Reduzierung des öffentlichen Spielraums resultiert. Es wird gesagt, dass Fachkräfte und Kinder über «Bücher und andere Medien» gemeinsam Fragen beantworten können. Der Plan schlägt ebenfalls Chatten und E-Mailverkehr mit einer Patengruppe vor, jedoch um das Interesse an geschriebener Sprache zu fördern, nicht spezifische Medienkompetenzen. Entsprechend kann hier eine schwache Verankerung von Medienpädagogik festgestellt werden.

\section{Eingeschränkte Verankerung}

Niedersachsen, Baden-Württemberg und Rheinland-Pfalz haben das Thema Medien eingeschränkt in ihren Plänen verankert.

Im niedersächsischen Plan wird der medienerzieherische Ansatz im Wesentlichen in nur einem Satz behandelt. Jedoch werden hier - wenn auch sehr knapp - zwei Dimensionen der Medienkompetenz, nämlich Medienkunde und Mediengestaltung, angesprochen: «Einige Tageseinrichtungen eröffnen Kindern auch einen altersgemässen Zugang zu technischen Medien, insbesondere dem Computer, damit sie dessen Lern- und Gestaltungsmöglichkeiten kennen lernen und kreativ nutzen können». Medienerziehung ist also optional; kritisch wird jedoch der Einsatz «rein rezeptive[r] PC-Programme» gesehen, diese würden das Kind nur «beschäftigen». Auch «übermäßiger Medienkonsum» (ausserhalb der Kita) wird problematisiert.

Der baden-württembergische Plan geht auch eingeschränkt auf medienerzieherische Themen ein, argumentiert allerdings, dass Medien für Kinder zum Alltag gehören und diese Erfahrungen verarbeitet werden müssen. Insgesamt wird Mediengebrauch jedoch im Spannungsverhältnis zu sinnlicher Erfahrung gesehen, weshalb es nicht verwunderlich ist, dass das Thema vor allem unter dem Bildungs- und Entwicklungsfeld «Sinne» behandelt wird.

Der rheinland-pfälzische Plan geht nicht auf die Dimension «Mediengestaltung» ein, weshalb die Verankerung als eingeschränkt angesehen werden kann. Dennoch muss hier angemerkt werden, dass der Plan für sein Erscheinungsdatum (2004) recht progressiv ist und auch als einziger der bisher aufgeführten Pläne (BB, ST, HB, NI, BW, SL, RP) Medien im Inhaltsverzeichnis und sogar als eigenen Bildungsbereich benennt. Wenngleich auch hier die Bedeutung von Primärerfahrungen im Vergleich zu (medial vermittelten) Sekundärerfahrungen betont wird, wird gleichzeitig festgestellt, dass Medien zur Kultur gehören und das Erschliessen dieser «eigene[n] Erfahrungsräume» ein «wichtiger Schritt in der Gesamtentwicklung der Kinder» darstellt. Der Plan unterstreicht: «[D]er souveräne Umgang mit ihnen [den Medien] gehört zu einer modernen Lebensgestaltung». 
Umfassende Verankerung

Zu den Plänen, die sich umfassend (aber nicht sehr umfassend) mit dem Thema Medien beschäftigen, also alle Dimensionen nach Baacke mindestens knapp ansprechen, gehören Mecklenburg-Vorpommern, Sachsen, Saarland, Berlin und Hamburg.

Im mecklenburg-vorpommerschen Plan tauchen Medien unter dem Punkt «Begegnung mit geschriebenen Texten und anderen Medien» im Inhaltsverzeichnis auf. Bereits hier zeigt sich, dass besonders (klassische) Schrift-/Printmedien gemeint sind, d.h. das Lesen und die Lesekultur stehen stark im Vordergrund. Der Umgang mit elektronischen oder digitalen Medien wird hingegen eher in der Familie erlernt. Die Dimensionen nach Baacke werden recht vereinzelt angesprochen, jedoch kaum näher ausgeführt und vorrangig auf den Umgang mit Texten bezogen. Der Plan hält «passiven Medienkonsum» für bildungs- und entwicklungshindernd und unterscheidet zwischen Primär- und Sekundärerfahrungen.

Im sächsischen Plan ist stellenweise eine hohe Technikskepsis zu erkennen (durch Techniknähe entstünden «künstliche, sterile und emotionslose Welten»), jedoch wird daraus geschlussfolgert, dass eine aktive Auseinandersetzung mit Medien notwendig ist. Hier wird die Kita in die Pflicht genommen, da viele Familien Unterstützung benötigen würden. So könne möglichen Chancenungleichheiten, die über den Umgang mit Medien verstärkt würden, entgegengewirkt werden. Der Plan legt zudem Wert auf einen - dem Entwicklungsstand entsprechenden - möglichst selbstständigen Umgang.

Der 2018 neu erschienene saarländische Plan weist im Vorwort darauf hin, Medienpädagogik nun stärker als zuvor zu integrieren. Dennoch wird das Verhältnis zu Medien und der damit verbundenen gesellschaftlichen Veränderung teilweise zwiespältig gesehen. ${ }^{18}$ So heisst es, Veränderungen wie «[...] der immer frühere Zugang von Kindern zu Medien aller Art eröffnen Kindern - ob wir das wollen oder nicht und ob sie das wollen oder nicht - neue Erfahrungshorizonte und bringen neue Zuordnungen». Medial vermittelte Situationen werden zwar als «künstlich» und «ungenügend» beschrieben, dennoch wird an anderer Stelle recht deutlich gefordert: «Die Kita darf sich diesen Erfahrungen nicht verschließen.» Ebenso wird betont, dass Medien selbstverständlich zum Alltag der Kinder dazugehören und bereits in der frühen Kindheit - entsprechend des Entwicklungsstands - Chancen für ein «lebenslanges und globales Lernen» böten; Kinder sollten daher nicht aus «falsch verstandene[r] Fürsorglichkeit» davon ferngehalten werden. Vielmehr sei ein eigenständiger Zugang zu fördern. Der Plan greift das Thema unter mehreren Bildungsbereichen in Form von (knappen) Fragen oder stichwortartigen Anregungen auf. Dabei wird auch der Einsatz von Tablets angesprochen.

18 Friedrichs-Liesenkötter (2019) geht gesondert auf den saarländischen Plan ein und hebt die dort vertretene medienskeptische Haltung als untypisch für die «grüne» Kategorisierung sowie die Aktualität des Plans hervor. 
Die Pläne von Berlin und Hamburg weisen relativ grosse Überschneidungen in Bezug auf Medien auf; die Textstellen zu «Entwicklung von Medienkompetenz» sind teilweise wortgleich. Die Ausführungen im Hamburger Plan sind etwas knapper als im Berliner, dafür aber insgesamt dem Medienthema gegenüber etwas aufgeschlossener. So betont der Berliner Plan, dass in der Kita reale Erfahrungen im Vordergrund stehen sollten, da die Kinder im familiären Umfeld ohnehin diverse Medien nutzen würden. Diese Passage fehlt im Hamburger Plan, während sich die deutliche Forderung «Die Auseinandersetzung mit Medienerfahrungen der Kinder als Teil ihrer Lebenswirklichkeit gehört deshalb in die Kita» nur dort findet. So wird auch im Berliner Plan das Thema Medien stärker über den Schutz vor Risiken hergeleitet, als über Chancen und Potenziale begründet. Jedoch fehlen im Hamburger Plan teilweise Beispiele zum Einsatz von Medien, sodass dieser etwas weniger konkret ist.

\section{Sehr umfassende Verankerung}

Sehr umfassend haben jene Pläne das Thema Medien und Medienerziehung verankert, die alle Dimensionen ansprechen und diese ausserdem besonders ausführlich behandeln. Zu diesen Bildungsplänen gehören Schleswig-Holstein, NRW, Thüringen, Hessen und Bayern.

Der schleswig-holsteinische Plan widmet sich Medien als Teil der musisch-ästhetischen Bildung; so heisst es im Titel des Kapitels «sich und die Welt mit allen Sinnen wahrnehmen». Entsprechend werden medial vermittelte Erfahrungen als «Erfahrungen aus zweiter Hand», beschrieben, welche sinnliche Erfahrungen ergänzen können. Auch hier wird auf die unterschiedlichen Erlebnisse, die Kinder zu Hause im Umgang mit Medien machen, hingewiesen - diese sollen in der Kita ausgeglichen und auch verarbeitet werden. Dies sollte entsprechend dem Entwicklungsstand der Kinder geschehen. In dem Textabschnitt zu Kindern im Alter von null bis drei Jahren spielt das Thema Medien jedoch keine Rolle. Die Dimensionen von Baacke werden ausführlich behandelt, die kritische Dimension wird allerdings vor allem in Bezug auf die reflexive Unterdimension angesprochen.

Der NRW-Plan führt «Medien» als einen von zehn Bildungsbereichen auf. Der Plan ist für die Arbeit mit Kindern von 0 bis 10 Jahre ausgelegt. Gefordert wird ein situationsorientierter und ganzheitlicher Ansatz, bei dem die Lebenswelt der Kinder im Mittelpunkt steht. Medien werden als «positive Erweiterung ihrer [der Kinder] sonstigen Erfahrungsmöglichkeiten» gesehen. Neben der Alltagsrelevanz von Medien wird auch auf ihre Bedeutung für eine «Kinderkultur» sowie die Möglichkeiten zur Teilhabe über Medien eingegangen. Um Chancenungleichheiten im Zusammenhang mit Medien auszugleichen, wird die Einbeziehung der Eltern und ihre systematische Unterstützung gefordert. 


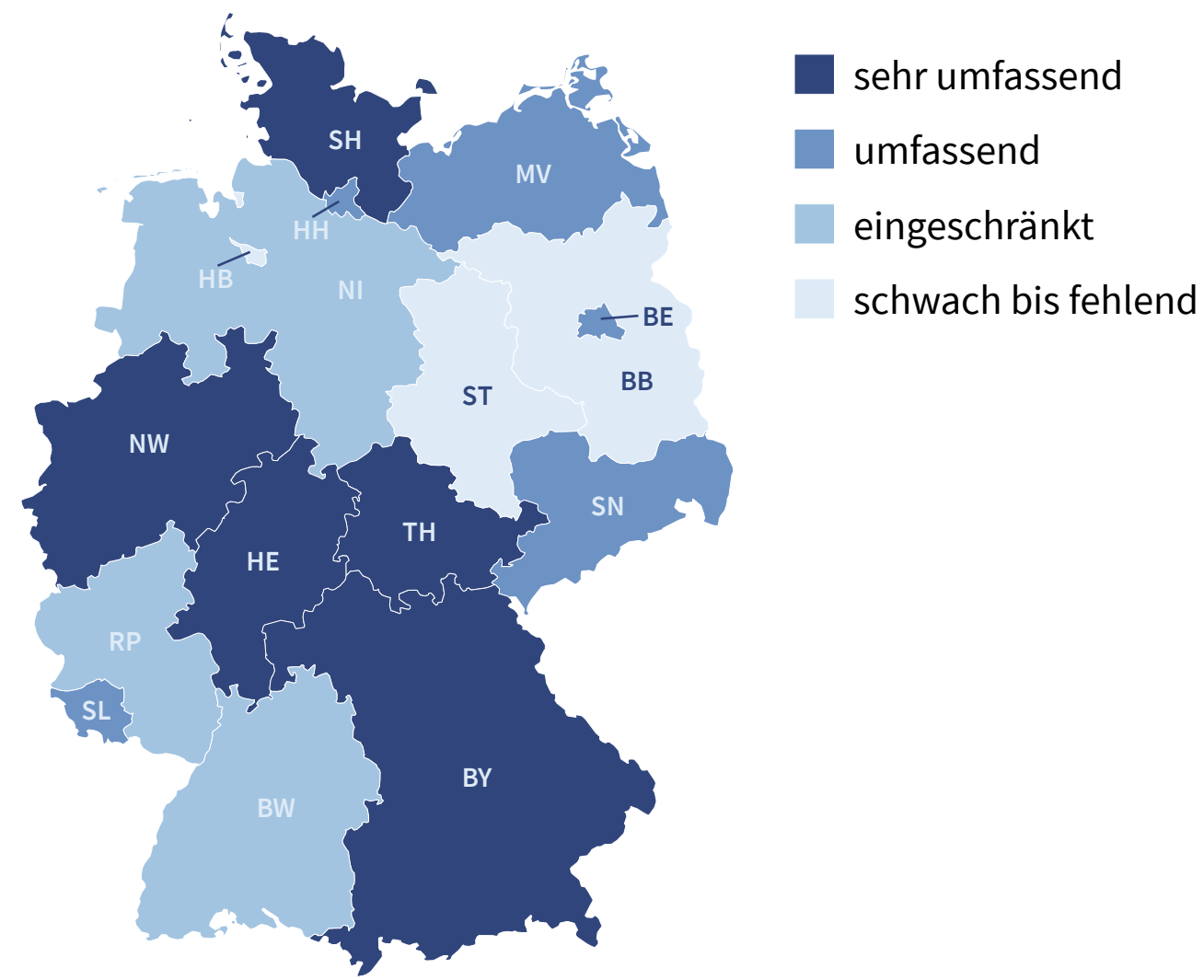

Abb. 1.: Intensität der Verankerung medienerzieherischer Inhalte in den Bildungsplänen der Bundesländer.

Der thüringische Plan fällt durch ein besonders differenziertes Medienverständnis auf und geht auf Themen wie Medienkonvergenz und die Besonderheiten im Umgang mit digitalen Medien gesondert ein. Medienbildung wird als lebenslanger Prozess und als Querschnittsaufgabe betrachtet, weshalb sie auch mit anderen Bildungsbereichen verknüpft wird. So wird deutlich formuliert: «Mehr als je zuvor ist erzieherisches und pädagogisches Handeln heute als Handeln in einer durch Medien geprägten Welt zu denken.» Um dies zu gewährleisten, weist der Plan auf eine thüringische Initiative hin, die seit 2010 vermehrt medienpädagogische Fortbildungen in Aus- und Weiterbildung von Pädagoginnen und Pädagogen verankert. Medienkompetenz wird nah an Baacke angelehnt, und es wird wissenschaftlich fundiert zwischen den verschiedenen Entwicklungsstufen differenziert.

Der hessische Plan überschneidet sich teilweise mit dem bayerischen Plan (beide wurden von Wassilios E. Fthenakis verfasst), wenngleich ersterer weniger umfangreich ist. So heben beide Pläne die Bedeutung von Medienkompetenz für die Teilhabe an der Gesellschaft besonders hervor (Medienkompetenz sei «unabdingbar»); ihr Erwerb soll im Wechselspiel zwischen Schutz und Befähigung zur Selbstständigkeit 
geschehen. Medienbildung soll zu mehr Chancengleichheit führen, weshalb damit bereits in der frühen Kindheit begonnen werden sollte (laut dem bayerischen Plan ist dies auch bei Kindern unter drei Jahren bereits im Ansatz möglich). Sie sollte dem Entwicklungsstand des Kindes entsprechen. Der bayerische Plan differenziert stärker zwischen unterschiedlichen Medien und behandelt auch die Verknüpfung dieser. Er betont, dass alle Medien hohe Lernpotenziale bieten. Wie diese genutzt werden können, schildert der bayerische Plan anhand vieler Praxisbeispiele.

Synthese: Intensität der Verankerung von Medienerziehung

Wie wir dargestellt haben, variiert die Intensität der Verankerung des Themas Medien bzw. Medienerziehung in den Bildungsplänen stark. So gibt es Pläne, die sich mit dem Thema nicht bzw. kaum auseinandersetzen, während andere seine besondere Bedeutung hervorheben oder vor diesem Hintergrund gar ein Umdenken im erzieherischen und pädagogischen Handeln fordern (TH).

Übergreifend lässt sich feststellen, dass die Pläne, die das Medienthema weniger vertiefend behandeln, ein Medienverständnis aufweisen, dass sich eher an klassischen, nicht-digitalen Medien orientiert (BB, BW, HB, SL, MV). Einige Pläne setzen mediale Erfahrungen in Opposition zu «realen» oder «sinnlichen» Erfahrungen (BE, BW, HE, MV, RP, SH, SL, SN). Ebenso gibt es Pläne, in denen hoher oder passiver Mediengebrauch bzw. -konsum problematisiert wird (BY, HB, MV, NI, SN, SH). In allen Plänen, die sich eingehender mit dem Thema befassen, wird auf die Alltagsrelevanz der Medien im Leben der Kinder hingewiesen (BE, BY, HE, HH, MV, SL, SH, SN, TH).

Die Pläne mit einer «sehr umfassenden» oder «umfassenden» Verankerung sprechen folgende Themen gehäuft an:

- dass Medienkompetenz oder -erziehung besonders wichtig ist (BY, HE, NW, RP, $\mathrm{TH}$ ),

- dass Medien ein Mittel für gesellschaftliche Teilhabe sind (BY, HE, NW, SN, TH),

- dass Medien wichtig für die Identitätsentwicklung der Kinder oder eine Kinderkultur sind (HH, NW, RP, SN, TH),

- dass Medien klischeehafte Darstellungen (etwa von Geschlechterrollen) propagieren (BE, HE, HH, SH, SL, TH),

- dass der divergierende Umgang mit Medien in der Familie Chancenungleichheiten verstärken kann (BY, HE, NW, SN, SH, SL, TH),

- dass Medienpädagogik an das Alter bzw. den Entwicklungsstand des Kindes angepasst sein sollte (BY, HE, SH, TH),

- dass Medienpädagogik sich an der Lebenswelt der Kinder orientieren sollte (BY, NW, TH),

- dass Medienpädagogik einen situationsorientierten Ansatz verfolgen (NW) bzW. von einem aktiven, souveränen Kind ausgehen sollte (BY, SN, NW, TH). 
Einzelne Pläne arbeiten mit praktischen Beispiele (BY, HE, NW, SH) oder differenzieren genauer zwischen verschiedenen Medien (BY, NW, TH). Der saarländische sowie der thüringische Plan, der für Kinder von 0 bis 18 Jahre verfasst ist, behandeln Medienbildung als lebenslangen Prozess. Auch die Zusammenarbeit mit den Eltern wird teilweise im Kontext des Themas Medien knapp angesprochen (BE, BW, BY, NW, SN, $\mathrm{TH}$ ), vereinzelt wird auch die Vorbildrolle von Erwachsenen in Bezug auf den Mediengebrauch betont (NW, TH).

Anhand der ausführlichen Auswertung wird das breite Spektrum der Herangehensweisen an das Thema Medien deutlich und es zeigen sich die verschiedenen inhaltlichen Schwerpunkte der Bundesländer. Die Bildungspläne behandeln das Thema keinesfalls nach "Schema F», sondern zeigen sehr unterschiedliche Haltungen. Wenngleich in dem sehr knapp gehaltenen «Gemeinsamen(r) Rahmen der Länder für die frühe Bildung in Kindertageseinrichtungen» Medienbildung angesprochen wird, sehen nicht alle Bildungspläne die Kita als Ort, an dem Kinder mit digitalen Medien umfassendere Erfahrungen machen sollen. Dennoch findet das Thema in der grossen Mehrzahl der Pläne Beachtung, wenngleich in unterschiedlicher Intensität. Von 16 Plänen befassen sich neun umfassend oder sehr umfassend mit Medien und haben entsprechend ein breites Verständnis von Medienkompetenz, wohingegen in nur zwei Plänen keine im Umgang mit Medien zu erlernenden Kompetenzen genannt werden. Darüber hinaus haben wir ausgeführt, dass die Pläne eine Vielzahl verschiedener Kompetenzen aufführen. Diese konnten anhand der vier Dimensionen des Medienkompetenzmodells nach Baacke strukturiert werden. An der Vielzahl dieser Kompetenzen lassen sich zweierlei Dinge erkennen: Erstens zeigt sich erneut, dass in der überwiegenden Mehrzahl der Bildungspläne die Meinung vertreten wird, dass Kinder bereits in der Kita umfassende Erfahrungen mit Medien machen können. Zweitens wird deutlich erkennbar, dass Medienerziehung nicht nur auf den Erwerb einer singulären Medienkompetenz abzielt, sondern dass der kompetente Umgang mit Medien diverse Kompetenzen umfasst. Von diesen können - so die Aussage der meisten Bildungspläne - viele bereits im Kita-Alter angestossen werden, entsprechend dem Entwicklungsstand des einzelnen Kindes. 


\begin{tabular}{|l|l|l|l|}
\hline Bundesland & $\begin{array}{l}\text { Erscheinungsjahr } \\
\text { des aktuellen } \\
\text { Plans }\end{array}$ & $\begin{array}{l}\text { Meister et al. } \\
(2012)\end{array}$ & Aktuelle Studie \\
\hline \multicolumn{2}{|l|}{ Seit der Analyse von Meister et al. (2012) in veränderter Neuauflage erschienene Pläne } \\
\hline Bayern & 2016 & \multicolumn{1}{|l|}{ umfassend } & sehr umfassend \\
\hline Berlin & 2014 & eingeschränkt & umfassend \\
\hline Hamburg & 2012 & umfassend & umfassend \\
\hline Hessen & 2016 & umfassend & sehr umfassend \\
\hline $\begin{array}{l}\text { Nordrhein- } \\
\text { Westfalen }\end{array}$ & 2016 & umfassend & sehr umfassend \\
\hline Saarland & 2018 & eingeschränkt & umfassend \\
\hline Sachsen & 2011 & umfassend & umfassend \\
\hline $\begin{array}{l}\text { Sachsen- } \\
\text { Anhalt }\end{array}$ & 2013 & eher schwach & fehlend bis schwach \\
\hline $\begin{array}{l}\text { Schleswig- } \\
\text { Holstein }\end{array}$ & 2012 & umfassend & sehr umfassend \\
\hline Thüringen & 2015 & eingeschränkt & sehr umfassend \\
\hline Seit der Analyse von Meister et al. (2012) unverändert gebliebene Pläne & \\
\hline $\begin{array}{l}\text { Baden- } \\
\text { Württemberg }\end{array}$ & 2011 & /19 & eingeschränkt \\
\hline Brandenburg & 2006 & eher schwach & fehlend bis schwach \\
\hline Bremen & $2012(2004)^{20}$ & eher schwach & fehlend bis schwach \\
\hline $\begin{array}{l}\text { Mecklenburg-Vorpom- } \\
\text { mern }\end{array}$ & 2011 & umfassend \\
\hline Niedersachsen & $2018(2005)^{21}$ & eingeschränkt \\
\hline Rheinland-Pfalz & $2016(2004)^{22}$ & eingeschränkt \\
\hline
\end{tabular}

Tab. 2.: Intensität der Verankerung von Medienkompetenz: Vergleich der Einordnung durch Meister et al. (2012) sowie der aktuellen Studie.

\section{Diskussion und Fazit}

In dieser Studie haben wir die Bildungspläne der 16 deutschen Bundesländer im Hinblick auf ihre Aussagen zu Medien, Medienerziehung und Medienbildung analysiert. Daraus hat sich ein nuanciertes Bild ergeben, welches - übereinstimmend mit Friedrichs-Liesenkötter (2019) - deutlich macht, dass die meisten Bundesländer dem

19 Der baden-württembergische Plan wird bei Meister et al. (2012) nicht eingeordnet.

20 Die Auflage von 2012 ist unverändert.

212018 ist der niedersächsische Bildungsplan als Gesamtausgabe mit den ergänzenden Handlungsempfehlungen erschienen. Der Bildungsplan ist jedoch unverändert.

22 Die Auflage von 2016 ist unverändert.

23 Der rheinland-pfälzische Plan taucht bei Meister et al. (2012) doppelt auf und wird, wohl fälschlicherweise, ebenfalls unter die Pläne mit eingeschränkter Verankerung eingeordnet. 
"Gemeinsamen[r] Rahmen der Länder für die frühe Bildung in Kindestageseinrichtungen» insofern folgen, als sie Medienerziehung in der frühkindlichen Bildung vorsehen, wenn auch in sehr unterschiedlicher Intensität. Wir konnten zeigen, dass in den meisten Ländern der (politische) Wille, Medienerziehung auch im frühpädagogischen Bereich zu integrieren, vorhanden ist und sich insbesondere die neueren Pläne ausführlich mit dem Thema auseinandersetzen. In Kontrast zu der Arbeit von Friedrichs-Liesenkötter (2019) schlagen wir eine vier- statt dreistufige Kategorisierung vor. Diese fusst auf inhaltlichen statt formalen Kriterien, wodurch eine (aus unserer Sicht notwenige) stärkere Ausdifferenzierung ermöglicht wird. Die eingehende Analyse der einzelnen Pläne bietet darüber hinaus einen Überblick zu den divergierenden Ansätzen der verschiedenen Bundesländer.

Ebenfalls konnten wir darlegen, welche Kompetenzen laut der Pläne in Bezug auf den Bildungsbereich Medien erworben werden sollten. Wenig überraschend (aber dennoch kritisch zu betrachten) wurde am häufigsten eine Nutzungs- und Bedienungskompetenz gefordert. Häufig bleiben die Pläne in Bezug auf die in der pädagogischen Arbeit zu vermittelnden Kompetenzen recht allgemein und schlagwortartig.

Dabei hat sich die Vorgehensweise, die Intensität der Verankerung medienerzieherischer Inhalte anhand von Baackes Medienkompetenzmodell zu bemessen, als überwiegend zielführend erwiesen. Ausnahmen sind die Pläne von Rheinland-Pfalz und Mecklenburg-Vorpommern: Während ersterer das Thema recht umfassend behandelt, aber nicht alle Dimensionen bespricht, widmet sich letzterer kaum den digitalen Medien, nennt dabei jedoch alle Dimensionen (wenn auch sehr knapp). Eine reine Einordnung anhand von Baackes Modell spiegelt also nicht immer den Stellenwert wider, den Medien im Plan einnehmen. Indem wir aber alle Pläne nicht nur eingruppieren, sondern auch einzeln besprechen, können wir hier stärker ausdifferenzieren und die unterschiedlichen Ansätze genauer beleuchten.

Aus unseren Ergebnissen lässt sich (übereinstimmend mit Friedrichs-Liesenkötter (2019)) ableiten, dass die neueren Pläne dem Thema Medienerziehung einen grösseren Stellenwert zuschreiben. So haben wir die Verankerung von Medienerziehung bei neun von den zehn Plänen, die seit der Datenerhebung durch Meister et al. (2012) neu erschienen sind, als umfassend oder sehr umfassend beschrieben. Hingegen wurden fünf der sechs Pläne, die vorher erschienen sind, von uns unter die Kategorien fehlende bis schwache bzw. eingeschränkte Verankerung eingeordnet. Auch im Vergleich der Ergebnisse von Meister et al. (2012) mit denen der vorliegenden Analyse zeigt sich, dass einige Bundesländer, die das Thema vorher nur schwach in den Plänen verankert hatten, nun offenbar stärker in den Fokus rücken (etwa BE, SL, TH). Obwohl das Thema Medien demnach in den Bildungsplänen in den vergangenen Jahren mehr Aufmerksamkeit erfährt, haben wir gezeigt, dass der Medienbegriff häufig undifferenziert gebraucht wird und insbesondere mobile digitale Medien kaum oder nur randständig behandelt werden. Hier herrscht dringender Handlungsbedarf. 
Wenngleich die meisten Pläne medienerzieherische Inhalte in der Kita fordern, kann allerdings nicht davon ausgegangen werden, dass dies in der Praxis bereits flächendeckend umgesetzt wird. Eine Umfrage der Stiftung Haus der kleinen Forscher aus dem Jahr 2017 ergab, dass nicht einmal in jeder fünften Kita der Einsatz und Umgang mit digitalen Medien im Konzept geregelt ist (17). Die MoFam-Studie besagt zudem, dass in den meisten Einrichtungen digitale Medien im pädagogischen Alltag nur eine kleine Rolle spielen und andere Erziehungsbereiche vielfach als relevanter angesehen werden (Schubert et al. 2018, 21).

Es ist fraglich, inwiefern die Bildungspläne ein geeignetes Mittel sind, um das Thema Medien stärker in den Fokus der frühkindlichen Bildung zu rücken. Manche Bundesländer bezeichnen ihren Bildungsplan zwar als verbindlich (siehe oben), jedoch haben diese Aussagen rechtlich wenig Wert, da hierfür oft die rechtliche Grundlage fehlt (Diskowski 2009). Selbst in Bundesländern, die ihren Bildungsplan als verbindlich definieren, sind nach unseren Informationen keine entsprechenden Prüfungsverfahren etabliert. Nach Auskunft des Berliner Kita-Instituts für Qualitätsentwicklung (welches die Implementierung des Berliner Bildungsprogramms unterstützt), ist Berlin das einzige Bundesland, das sich zu einer Evaluation verpflichtet hat.

Welche Rolle spielen also die Bildungspläne in der Praxis der frühkindlichen Bildungseinrichtungen? Es ist bislang unklar, inwiefern die Intensität der Verankerung des Medienthemas im Bildungsplan (ob als eigenständiger oder nicht-eigenständiger Bildungsbereich) Einfluss auf den Stellenwert im pädagogischen Alltag der Kitas nimmt. Meister et al. (2012) stellten im Rahmen einer Befragung von Kitas in NRW (vornehmlich Kita-Leitungen) heraus, dass ein Viertel der Befragten noch nicht einmal vom damaligen Bildungsplan gehört hatte (25). Viernickel et al. (2013) konnten drei Typen von Fachkräfte-Teams in Bezug auf ihre Umsetzung der Bildungspläne ausmachen. Die Haltungen reichen dabei von Ablehnung und Distanz bis zu einer (über)korrekten Abarbeitung der formulierten Anforderungen. Insofern ist die Aussage von Diskowksi (2009) zumindest teilweise zu relativieren, demzufolge «die erforderlichen tiefgreifenden Veränderungen in den Haltungen und Handlungen der Fachkräfte [...] aber durch Bildungspläne und andere normative Vorgaben nicht bewirkt werden [können]» (56). Er schreibt ihnen jedoch «eine wichtige stützende, legitimierende und normierende Funktion» zu (ebd.). Diese Einschätzung stützt auch ein Gutachten des ifib (2015); sie schreiben, dass «vorrangig dort adressierte Bereiche [...] eine Chance auf langfristige Förderung durch Landesprogramme [haben]» (ebd., 39). Vor diesem Hintergrund ist die Forderung zu sehen, dass Medien als eigenständiger Bildungsbereich in die Pläne integriert werden sollten (Kammerl und Thumel 2015, 9). Unsere Analyse stützt allerdings nicht, dass eine solche Eigenständigkeit Voraussetzung für eine umfassende Verankerung des Themas in den Bildungsplänen ist. Es wäre jedoch zu erforschen, ob in den Bundesländern, in denen diese Forderung 
umgesetzt wurde, die Relevanz des Bereichs Medien unter pädagogischen Fachkräften grössere Zustimmung erfährt. Mit der vorliegenden Studie ergänzen wie die Arbeit von Friedrichs-Liesenkötter (2019) und bieten wir eine Grundlage für solche und weitere Untersuchungen.

\section{Literatur}

Anfang, Günther. 2018. «Chancen der Digitalisierung im Bildungssystem Kita. Günther Anfang im Gespräch mit Eva Reichert-Garschhammer». merz medien + erziehung. Kita digital: Frühe Medienerziehung, Nr. 2.

Aufenanger, Stefan. 1999. «Medienkompetenz oder Medienbildung? Wie die neuen Medien Erziehung und Bildung verändern». Bertelsmann Briefe 142: 21-24.

Baacke, Dieter. 2007. Medienpädagogik. Nachdr. Grundlagen der Medienkommunikation 1. Tübingen: Niemeyer.

Betz, Tanja. 2013. «Anforderungen an Fachkräfte in Kindertageseinrichtungen». In Handbuch frühkindliche Bildungsforschung, herausgegeben von Margrit Stamm und Doris Edelmann, 259-72. Wiesbaden: Springer Fachmedien Wiesbaden. https://doi.org/10.1007/978-3-53119066-2_18.

Brüggemann, Marion, Ines Averbeck, und Andreas Breiter. 2013. «Förderung von Medienkompetenz in Bremer Kindertageseinrichtungen». Bremen. https://www.bremische-landesmedienanstalt.de/uploads/Texte/Meko/Forschung/Meko-Kita-Sept2013_ifib\%20-\%20FINAL. pdf.

Council of Europe. 2019. «Leitlinien zur Achtung, zum Schutz und zur Verwirklichung der Rechte des Kindes im digitalen Umfeld. Empfehlung CM/Rec(2018)7 des Ministerkomitees an die Mitgliedstaaten». https://edoc.coe.int/en/children-and-the-internet/7922-leitlinien-zurachtung-zum-schutz-und-zur-verwirklichung-der-rechte-des-kindes-im-digitalen-umfeldempfehlung-cmrec20187-des-ministerkomitees-an-die-mitgliedstaaten.html.

Diskowski, Detlef. 2009. «Bildungspläne für Kindertagesstätten - ein neues und noch unbegriffenes Steuerungsinstrument». In Frühpädagogische Förderung in Institutionen, herausgegeben von Hans-Günther Roßbach und Hans-Peter Blossfeld, 47-61. Wiesbaden: VS Verlag für Sozialwissenschaften. https://doi.org/10.1007/978-3-531-91452-7_4.

Eder, Sabine, Marion Brüggemann, und Jörg Kratzsch. 2017. «Kinder im Mittelpunkt: Frühe Bildung und Medien gehören zusammen. Positionspapier der GMK-Fachgruppe Kita». https:// www.gmk-net.de/fileadmin/pdf/gmk_medienbildung_kita_positionspapier.pdf.

Eder, Sabine. 2018. «Die frühe Medienpädagogik steht vor große Herausforderungen. Ein Interview mit Sabine Eder, Blickwechsel e.V.». Interviewt von Anfang, Günther. Merz. medien + erziehung 622.

Eggert, Susanne, und Ulrike Wagner. 2016. «Grundlagen zur Medienerziehung in der Familie. Expertise im Rahmen der Studie MoFam - Mobile Medien in der Familie.» http://www.jff. de/studie_mofam. 
Feierabend, Sabine, Theresa Plankenhorn, und Thomas Rathgeb. 2015. «miniKIM 2014. Kleinkinder und Medien. Basisuntersuchung zum Medienumgang 2-5-Jähriger in Deutschland». Herausgegeben von Medienpädagogischer Forschungsverbund Südwest. https://www. mpfs.de/fileadmin/files/Studien/miniKIM/2014/Studie/miniKIM_Studie_2014.pdf.

Feierabend, Sabine, Theresa Plankenhorn, und Thomas Rathgeb. 2017a. «FIM-Studie 2016. Familie, Interaktion, Medien. Untersuchung zur Kommunikation und Mediennutzung in Familien». Stuttgart. https://www.mpfs.de/fileadmin/files/Studien/FIM/2016/FIM_2016_ PDF_fuer_Website.pdf.

Feierabend, Sabine, Theresa Plankenhorn, und Thomas Rathgeb. 2017b. «KIM-Studie 2016. Kindheit, Internet, Medien. Basisstudie zum Medienumgang 6- bis 13-Jähriger in Deutschland». Stuttgart. https://www.mpfs.de/fileadmin/files/Studien/KIM/2016/KIM_2016_WebPDF.pdf.

Fleischer, Sandra, Peter Kroker, und Susanne Schneider. 2018. «Medien, frühe Kindheit und Familie». In Frühe Kindheit und Medien - Aspekte der Medienkompetenzförderung in der Kita, herausgegeben von Jürgen Georg Brandt, Manfred Kaulbach, Christine Hoffmann, und Thomas Schmidt, 35-52. Opladen Berlin Toronto: Verlag Barbara Budrich.

Friedrichs, Henrike, und Dorothee M. Meister. 2015. «Medienerziehung in Kindertagesstätten - Nachhaltigkeitsüberlegungen Im Anschluss an Eine Fortbildungsinitiative». MedienPädagogik: Zeitschrift für Theorie Und Praxis Der Medienbildung 22 (Frühe Medienbildung), 1-23. https://doi.org/10.21240/mpaed/22/2015.05.28.X.

Friedrichs-Liesenkötter, Henrike. 2019. «,Wo Medienbildung draufsteht, steckt nicht unbedingt Medienbildung drin' Eine Dokumentenanalyse von Bildungsplänen und Curricula in Ausbildung und Studium zur frühkindlichen Medienbildung und -erziehung», Medienimpulse, 57(1). https://journals.univie.ac.at/index.php/mp/article/view/mi1318.

Guth, Birgit. 2018. «Vorschulkinder im Fokus. Mediennutzung bei den Kleinen». https://www. ip.de/lp/download_pdf.cfm?objectld=68910.

Hepp, Andreas. 2018. «Von der Mediatisierung zur tiefgreifenden Mediatisierung. Konstruktivistische Grundlagen und Weiterentwicklungen in der Mediatisierungsforschung». In Kommunikation - Medien - Konstruktion. Braucht die Mediatisierungsforschung den Kommunikativen Konstruktivismus?, herausgegeben von J Reichertz und R Bettmann, 27-45. Wiesbaden: Springer VS. https://doi.org/10.1007/978-3-658-21204-9_2.

Institut für Demoskopie Allensbach, Hrsg. 2014. «Digitale Medienbildung in Grundschule und Kindergarten. Ergebnisse einer Befragung von Eltern, Lehrkräften an Grundschulen und Erzieher(innen) in Kindergärten im Auftrag der Deutsche Telekom Stiftung». https://www. telekom-stiftung.de/sites/default/files/files/media/publications/ergebnisse_allensbachumfrage_gesamt.pdf.

Institut für Informationsmanagement Bremen GmbH (ifib), Hrsg. 2015. «Zugangs- und Nutzungsbedingungen als substantielle Voraussetzung zur Wahrnehmung von Bildungschancen.»http://www.ifib.de/publikationsdateien/tab_gutachten.pdf. 
Jörissen, Benjamin, und Winfried Marotzki. 2009. Medienbildung - eine Einführung: Theorie Methoden - Analysen. UTB Erziehungswissenschaft, Medienbildung 3189. Bad Heilbrunn: Klinkhardt.

Jugendministerkonferenz, Kultusministerkonferenz. 2004. «Gemeinsamer Rahmen der Länder für die frühe Bildung in Kindertageseinrichtungen». https://www.kmk.org/fileadmin/Dateien/veroeffentlichungen_beschluesse/2004/2004_06_03-Fruehe-Bildung-Kindertageseinrichtungen.pdf.

Kammerl, Rudolf, und Mareike Thumel. 2016. «Medienpädagogik in der Kita. Eine Expertise zum Stand in Hamburg und Schleswig-Holstein im Auftrag der Medienanstalt Hamburg / Schleswig-Holstein (MA HSH)». https://www.ma-hsh.de/infothek/publikationen/medienkompetenz-expertisen.html?file=files/infothek/publikationen/Medienpaedagogik\%20 in\%20der\%20Kita\%202018/Medienpaedagogik_Kita_web.pdf.

Meister, Dorothee M., Henrike Friedrichs, Karolina Keller, Anja Pielsticker, und Timon Tobias Temps. 2012. «Chancen und Potenziale digitaler Medien zur Umsetzung des Bildungsauftrags in Kindertageseinrichtungen in NRW. Forschungsbericht der Gesellschaft für Medienpädagogik und Kommunikationskultur (GMK) und der Universität Paderborn». Forschungsbericht der Gesellschaft für Medienpädagogik und Kommunikationskultur (GMK) und der Universität Paderborn. Paderborn. https://kw.uni-paderborn.de/fileadmin/fakultaet/Institute/medienwissenschaften/Medienpaedagogik_und_empirische_Medienforschung/Archiv/Kitas_NRW_bericht_2012.pdf.

Neuß, Norbert. 2013. «Medienkompetenz in der frühen Kindheit.»In Medienkompetenzförderung für Kinder und Jugendliche - Eine Bestandsaufnahme, herausgegeben vom Bundesministerium für Familie, Senioren, Frauen und Jugend, 34-45. https://www.gmk-net.de/wpcontent/uploads/2018/07/medienkompetenzbericht_2013.pdf.

Roboom, Susanne. 2017. Mit Medien kompetent und kreativ umgehen: Basiswissen \& Praxisideen. 1. Auflage. Beltz Nikolo / Kita kompakt. Weinheim Basel: Beltz Nikolo.

Rosebrock, Cornelia, und Olga Zitzelsberger. 2002. «Der Begriff Medienkompetenz als Zielperspektive im Diskurs der Pädagogik und Didaktik.» In Medienkompetenz - Voraussetzungen, Dimensionen, Funktionen, hrsg. v. Norbert Groeben u. Bettina Hurrelmann, 148-159. Weinheim: Juventa.

Schorb, Bernd. 2009. «Gebildet und kompetent. Medienbildung statt Medienkompetenz?» merz: Medien + Erziehung 53 (5): 50-56. https://www.lmz-bw.de/fileadmin/user_upload/ Downloads/Handouts/schorb-gebildet-und-kompetent.pdf.

Sektion Medienpädagogik, DGfE. 2017. «Orientierungsrahmen für die Entwicklung von Curricula für medienpädagogische Studiengänge und Studienanteile». MedienPädagogik: Zeitschrift für Theorie und Praxis der Medienbildung, (Einzelbeiträge). https://doi.org/10.21240/ mpaed/00/2017.12.04.x.

Spanhel, Dieter. 2002. «Medienkompetenz als Schlüsselbegriff der Medienpädagogik?». In forum medienethik (Vol. 1): 48-53. München: kopaed. https://www.lmz-bw.de/fileadmin/ user_upload/Downloads/Handouts/spanhel-medienkompetenz-schluesselbegriff-medienpaedagogik.pdf. 
Spanhel, Dieter. 2010. «Medienbildung statt Medienkompetenz? Zum Beitrag von Bernd Schorb (merz 5/09).» merz: Medien + Erziehung 54 (1): 49-54.

Spanhel, Dieter. 2011. «Medienbildung als Grundbegriff der Medienpädagogik. Begriffliche Grundlagen für eine Theorie der Medienpädagogik». MedienPädagogik: Zeitschrift für Theorie und Praxis der Medienbildung, September, 95-120. https://doi.org/10.21240/ mpaed/20/2011.09.15.x.

Statistisches Bundesamt (Destatis). 2018. «Kinder und tätige Personen in Tageseinrichtungen und in öffentlich geförderter Kindertagespflege am 01.03.2018.» https://www.destatis.de/ DE/Publikationen/Thematisch/Soziales/KinderJugendhilfe/TageseinrichtungenKindertagespflege5225402187004.pdf?__blob=publicationFile.

Stiftung Haus der kleinen Forscher, Hrsg. 2017. «,Wie nutzen Erzieherinnen und Erzieher digitale Geräte in Kitas?‘ - Eine repräsentative Telefonumfrage». https://www.haus-der-kleinen-forscher.de/fileadmin/Redaktion/3_Aktuelles/Presse/171213_Ergebnisse_zur_Telefonbefragung_Digitales.pdf.

Textor, Martin R. 2008. «Erziehungs- und Bildungspläne». http://kindergartenpaedagogik.de/ fachartikel/bildung-erziehung-betreuung/1951.

Thomann, Marius. 2015. «Medienkompetenz oder Medienbildung? Zur Frage nach dem Zielwert medienpädagogischer Praxis». MedienPädagogik: Zeitschrift für Theorie und Praxis der Medienbildung, (Einzelbeiträge). https://doi.org/10.21240/mpaed/00/2015.02.23.x.

Tilemann, Friederike. 2018. «,Foto, Film und Wachsmalstift‘ - Miedienpädagogik mit jungen Kindern». In Frühe Kindheit und Medien - Aspekte der Medienkompetenz in der Kita, herausgegeben von Jürgen Georg Brandt, Christine Hoffmann, Manfred Kaulbach, und Thomas Schmidt, 15-26. Opladen Berlin Toronto: Verlag Barbara Budrich.

Tulodziecki, Gerhard, Hrsg. 1995. Handlungsorientierte Medienpädagogik in Beispielen: Projekte und Unterrichtseinheiten für Grundschulen und weiterführende Schulen. Bad Heilbrunn: Klinkhardt.

Tulodziecki, Gerhard. 2008. «Medienerziehung». In Handbuch Medienpädagogik, herausgegeben von Uwe Sander, Friederike von Gross, und Kai-Uwe Hugger, 110-15. Wiesbaden: VS Verlag für Sozialwissenschaften. https://doi.org/10.1007/978-3-531-91158-8_12.

Tulodziecki, Gerhard. 2010. «Medienkompetenz und/oder Medienbildung? Ein Diskussionsbeitrag.» merz: Medien + Erziehung 54 (3): 48-53.

Tulodziecki, Gerhard. 2011. "Zur Entstehung und Entwicklung zentraler Begriffe bei der pädagogischen Auseinandersetzung mit Medien.» In Medienbildung und Medienkompetenz Beiträge zu Schlüsselbegriffen der Medienpädagogik, hrsg. v. Heinz Moser, Petra Grell u. Horst Niesyto, 11-39. München: kopaed. https://doi.org/10.21240/mpaed/20/2011.09.11.X.

Urlen, Marc. 2018. DJI-Projekt «Apps für Kinder» - Trendanalyse Nr. 3 Handlungsorientierte Medienpädagogik im digitalen Raum. Ansätze zur Vermittlung von Medienkompetenzen und zur Beurteilung von Kindersoftware. https://www.dji.de/fileadmin/user_upload/kinderapps/Apps_fuer_Kinder_Trendanalyse_3.pdf 
Viernickel, Susanne, Iris Nentwig-Gesemann, Katharina Nicolai, Stefanie Schwarz, und Luise Zenker. 2013. Schlüssel zu guter Bildung, Erziehung und Betreuung: Bildungsaufgaben, Zeitkontingente und strukturelle Rahmenbedingungen in Kindertageseinrichtungen. 1. Aufl. Forschungsbericht. Berlin: Der Paritätische Gesamtverb. http://docplayer.org/17635320Forschungsbericht-schluessel-zu-guter-bildung-erziehung-und-betreuung.html.

\section{Bildungspläne}

Senatsverwaltung für Bildung, Jugend und Wissenschaft, Hrsg. 2014. Berliner Bildungsprogramm für Kitas und Kindertagespflege. Aktualisierte Neuaufl. Weimar: verlag das netz. https://www.berlin.de/sen/jugend/familie-und-kinder/kindertagesbetreuung/berliner_bildungsprogramm_2014.pdf.

Hessisches Ministerium für Soziales und Integration, und Hessisches Kultusministerium, Hrsg. 2017. Bildung von Anfang an: Bildungs- und Erziehungsplan für Kinder von 0 bis 10 Jahren in Hessen. 7. Aufl., 2016. Wiesbaden: Hessisches Sozialministerium [u.a.]. https:// bep.hessen.de/sites/bep.hessen.de/files/content-downloads/Bildungs-und_Erziehungsplan_2016-09-23.pdf.

Ministerium für Bildung, Frauen und Jugend, Hrsg. 2004. Bildungs- und Erziehungsempfehlungen für Kindertagesstätten in Rheinland-Pfalz. https://kita.rlp.de/fileadmin/kita/04_Service/01_Gesetze__Verordnungen_Empfehlungen/3._Verordnungen_und_Empfehlungen/ BEE_Gesamt_geschuetzt_2019.pdf.

Ministerium für Familie, Kinder, Jugend, Kultur und Sport des Landes Nordrhein-Westfalen, Hrsg. 2016. Bildungsgrundsätze: mehr Chancen durch Bildung von Anfang an; Grundsätze zur Bildungsförderung für Kinder von 0 bis 10 Jahren in Kindertagesbetreuung und Schulen im Primarbereich in Nordrhein-Westfalen. Freiburg Basel Wien: Herder. https://www.kita. nrw.de/file/1708/download?token=jKG5KdQB.

Ministerium für Arbeit und Soziales des Landes Sachsen-Anhalt, Hrsg. 2014. Bildungsprogramm für Kindertageseinrichtungen in Sachsen-Anhalt: Bildung: elementar - Bildung von Anfang an; Fortschreibung 2013. 1. Aufl. Weimar Berlin: Verl. das netz. https://ms.sachsen-anhalt. de/fileadmin/Bibliothek/Politik_und_Verwaltung/MS/MS/Presse_Dialog_Kita/2014/bildungsprogramm_2014.pdf.

Ministerium für Bildung und Kultur Saarland, Hrsg. 2018. Bildungsprogramm mit Handreichungen für saarländische Krippen und Kindergärten. Weimar Berlin: Verl. Das Netz. https:// www.saarland.de/dokumente/thema_bildung/Saarland_Inhalt_2018_screen.pdf.

Bayerisches Staatsministerium für Arbeit und Sozialordnung und Familie und Frauen, und Staatsinstitut für Frühpädagogik München, Hrsg. 2016. Der Bayerische Bildungs- und Erziehungsplan für Kinder in Tageseinrichtungen bis zur Einschulung. 6. Aufl. Frühe Kindheit. Berlin: Cornelsen. https://www.ifp.bayern.de/imperia/md/content/stmas/ifp/bildungsplan_7._auflage.pdf. 
Sächsisches Staatsministerium für Kultus, Hrsg. 2012. Der sächsische Bildungsplan: ein Leitfaden für pädagogische Fachkräfte in Krippen, Kindergärten und Horten sowie für Kindertagespflege. Veränd. Neuauflage. Weimar Berlin: Verl. das Netz. https://www.kita-bildungsserver.de/wp-content/themes/kbs/inc/downloads_ausliefern.inc.php?did=37.

Ministeriums für Bildung, Wissenschaft und Kultur Mecklenburg-Vorpommern, Hrsg. 2011. Die Bildungskonzeption für 0- bis 10-jährige Kinder in Mecklenburg-Vorpommern zur Arbeit in Kindertageseinrichtungen und Kindertagespflege. https://www.bildung-mv.de/export/sites/bildungsserver/downloads/Bildungskonzeption_0bis10jaehrige.pdf.

Ministerium für Soziales, Gesundheit, Wissenschaft und Gleichstellung des Landes SchleswigHolstein, Hrsg. 2012. Erfolgreich starten - Leitlinien zum Bildungsauftrag in Kindertageseinrichtungen. https://www.schleswig-holstein.de/DE/Fachinhalte/K/kindertageseinrichtungen/downloads/kindertageseinrichtungen_Bildungsauftrag_LeitlinienBildungsauftrag_ BildungsauftragLeitlinien.pdf?_blob=publicationFile\&v=1.

Ministerium für Bildung, Jugend und Sport Land Brandenburg, Hrsg. 2006. Grundsätze elementarer Bildung in Einrichtungen der Kindertagesbetreuung im Land Brandenburg. https:// mbjs.brandenburg.de/media/lbm1.c.312232.de.

Behörde für Arbeit, Soziales, Familie und Integration Freie und Hansestadt Hamburg, Hrsg. 2012. Hamburger Bildungsempfehlungen für die Bildung und Erziehung von Kindern in Tageseinrichtungen. Zweite überarbeitete Neuauflage. https://www.hamburg.de/contentblo b/118066/2a650d45167e815a43999555c6c470c7/data/bildungsempfehlungen.pdf.

Ministerium für Kultus, Jugend und Sport Baden-Württemberg, Hrsg. 2011. Information zum Orientierungsplan für Bildung und Erziehung in baden-württembergischen Kindergärten und weiteren Kindertageseinrichtungen. http://kindergaerten-bw.de/site/pbs-bw-new/get/ documents/KULTUS.Dachmandant/KULTUS/Projekte/kindergaerten-bw/Oplan/Material/ KM-KIGA_Orientierungsplan_2011.pdf?attachment=true.

Niedersächsisches Kultusministerium, Hrsg. 2005. Orientierungsplan für Bildung und Erziehung im Elementarbereich niedersächsischer Tageseinrichtungen für Kinder. https://www. mk.niedersachsen.de/download/4491. 